\title{
Effective osmotic cohesion due to the solvent molecules in a delocalized adsorbed monolayer
}

\author{
Radomir I. Slavchov ${ }^{1}$, Ivan B. Ivanov ${ }^{2}$ \\ ${ }^{1}$ Department of Chemical Engineering \\ University of Cambridge \\ ${ }^{2}$ Laboratory of Chemical Physics \\ and Engineering \\ West Site, Philippa Fawcett Drive \\ Faculty of Chemistry and Pharmacy \\ Cambridge, CB3 OAS \\ United Kingdom \\ Sofia University \\ E-mail: ris26@cam.ac.uk \\ $1 \mathrm{~J}$. Bourchier, 1164 Sofia, Bulgaria
}

\begin{abstract}
An equation of state for an uncharged delocalized surfactant monolayer adsorbed at a liquid interface is derived, taking explicit account for the solvent molecules present in the monolayer. The model is based on the scaled particle theory of hard-disc mixtures, and is also extended to sticky discs (i.e. attraction between the adsorbed molecules). The osmotic effect due to the solvent in the adsorbed layer is shown to be equivalent to an effective lateral attraction between the surfactant molecules. This effective osmotic cohesion causes an increase of the value of the attraction parameter $\beta$ of the monolayer. The smaller the size of the surfactant polar head group is, the larger the effective attraction the model predicts. This trend is verified with data for the adsorption at water|air surface of alcohols, undissociated acids, and hexaethylenglycol monoalkyl ethers. The proposed theory allows the amount of solvent in the monolayer to be estimated.
\end{abstract}

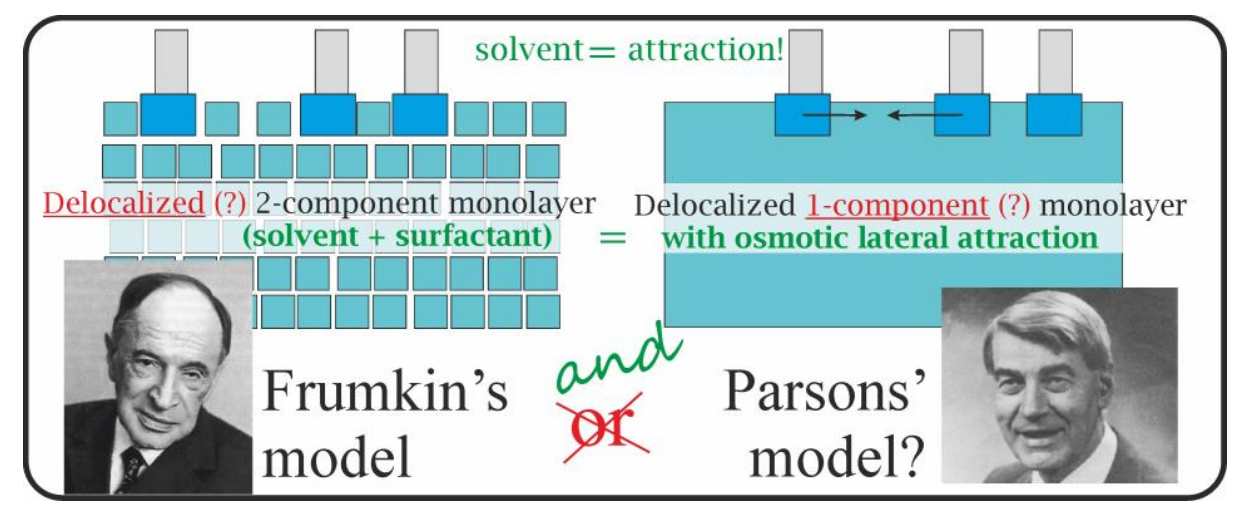

Keywords: adsorption, liquid interface, osmotic effect, cohesion, monolayer, non-ionic surfactant, ethylenglycol monoalkyl ethers, alcohols 


\section{Introduction 1}

Half a century has passed since the famous scientific "battle" between Frumkin and his collaborators, and Parsons, Buff and Stillinger. The battlefield was the theory of adsorption [1]. Parsons, Buff and Stillinger [2-4] argued that a site model such as Langmuir's cannot provide a satisfactory description for the strictly delocalized adsorption of ions at the water|mercury interface; they have championed instead the theory of two dimensional (2D) hard-disc liquids and a nearly exact equation of state (EoS) due to Helfand, Frisch, and Lebowitz [5] (HFL). In response, Frumkin $[1,6]$ reminded that the Langmuir equation, when applied to adsorption of ions from aqueous solution, is not merely a surface site model - it is a direct consequence of the lattice (Flory-Huggins) theory for a mixture of water and ions. Frumkin further pointed out that a single component EoS such as HFL, with its complete neglect of the water molecules, is no sounder than the Langmuir model. The response of Parsons - then a young man having a great respect for his senior colleague [7] - to Frumkin's criticism was half-hearted [8].

The Langmuir model and its derivatives still dominate the adsorption literature [9-15]. Significantly, Parsons himself was not persistent in pursuing a delocalized description of the adsorbed layer and, in his later works, he worked on localized models for liquid interfaces, e.g., [16]. This might seem as a victory for Frumkin, but Frumkin himself was not too convinced either, stating that his arguments "cannot serve, of course, as a sufficient theoretical basis for the application of Langmuir's equation to real systems... but it seems to me that these arguments can help when choosing the direction for the further development of the theory of adsorption at the surface of solutions" [6]. Occasionally, variants of the HFL EoS are used for surfactant monolayers [17-23], and indeed have clear advantages. In fact, the application of the Langmuir model (or its extensions to cohesive monolayers, such as Frumkin's model) to surfactant films adsorbed at liquid interfaces leads to several paradoxes which can be traced back to the delocalized nature of the monolayer [23]:

(i) the Langmuir model's area per molecule determined from adsorption data is as much as twice as large as the crystallographic one, while, in theory, the two quantities should have similar values.

(ii) The area parameter of Langmuir's model is not transferable from one type of interface to another - to fit the experimental data, one has to use one area of the surfactant for water|air $(\mathrm{W} \mid \mathrm{A})$ and another for water|oil interfaces.

(iii) Frumkin's model (localized EoS with attraction) modifies Langmuir's to account for $1^{\text {st }}$ neighbours' attraction via the lateral attraction parameter $\beta$. When applied to adsorption data for surfactants at water|oil interfaces, this model yields unphysical negative values of $\beta$, a paradox that disappears when Parsons' model (delocalized EoS with attraction) is used instead - the expected small positive $\beta$ are obtained with it.

In any case, the advantage of this or the other model when compared to experimental data does not change the fact that both sides in the dispute were most definitely correct: the Langmuir model and the lattice theory of adsorption provide a description of the adsorption layer that is localized, which is not realistic for liquid interfaces; and, indeed, the HFL model is unconvincing for it neglects completely the solvent molecules at the surface (the amount of solvent in the monolayer is essential for its properties, e.g., Ref. [24]).

The aim of this work is to resolve the dispute by providing a description that is both delocalized and accounts for the solvent. In other words, we propose a theory of the osmotic effect due to the solvent molecules present in a monolayer adsorbed at a liquid interface

\footnotetext{
${ }^{1}$ Days before the completion of this work, Professor I. B. Ivanov (1935-2018) has sadly passed away. His relentless passion for knowledge, eloquence, clarity of mind, and strength of character will always be an example to follow for me.
} 
(delocalized adsorption) on the thermodynamic properties of this monolayer. In Section 2.2, we analyse the theory of a 2-component hard-disc mixture (direct attraction neglected) and we show that the presence of solvent in the monolayer results in effective osmotic cohesion of it. In Section 2.3, we simplify the results for hard-disc mixture by making use of the concept for osmotic cohesion, and in Sections $2.3 \& 3$, we generalize it to strongly cohesive (attractive) molecules. The new model so-obtained is a natural theoretical approach to adsorption of nonionic amphiphiles at liquid interfaces. We demonstrate the feasibility of the model by comparing it to data for the adsorption at water|air of 3 homologous series of surfactants of different hard-disc area of the polar head group: alcohols, non-dissociated acids and hexaethyleneglycol monoalkyl ethers (Section 3).

The terminology we use in this manuscript is standard for the field of statistical and chemical thermodynamics (localized, delocalized, osmotic effect), but for the ease of readers of other backgrounds and to avoid confusion, these are described in the supplementary material $\mathrm{S} 1$; there, a list of symbols and abbreviations is also given.

\section{Theory}

\subsection{Single component hard-disc liquid}

Before approaching the problem of two-component monolayers, we will briefly review the theory of delocalized single component hard-disc 2D fluid. Helfand, Frisch and Lebowitz derived an almost exact surface EoS for delocalized adsorption layer of hard discs in the absence of attraction, by using the apparatus of the scaled particle theory [5]:

$$
\frac{\alpha_{\mathrm{s}} \pi^{\mathrm{S}}}{k_{\mathrm{B}} T}=\frac{\psi_{\mathrm{s}}}{\left(1-\psi_{\mathrm{s}}\right)^{2}} .
$$

Here, $\pi^{\mathrm{S}}$ is the surface pressure of the monolayer, $\pi^{\mathrm{S}} \equiv \sigma_{0}-\sigma, \sigma$ is surface tension; $\sigma_{0}$ is surface tension of the neat surface (at $\psi_{\mathrm{s}}=0$ ); $\psi_{\mathrm{s}} \equiv \alpha_{\mathrm{s}} \Gamma_{\mathrm{s}}$ is the surface fraction covered by surfactant; $\Gamma_{\mathrm{s}}$ is adsorption of surfactant; $\alpha_{\mathrm{s}}$ is the hard-disc area of the surfactant molecule. The HFL model has been found to agree excellently with data for monolayers of both ionic and non-ionic surfactants at water|oil interfaces [22,23], where other popular models lead to unreasonable adsorption parameters. The hard-disc (repulsion only) HFL model is not suitable for W|A, as at this interface there is a significant lateral van der Waals attraction between the adsorbed molecules. Parsons [3] generalized the HFL EoS (1) to attractive molecules by adding to it a binary interaction term, $\beta \psi_{\mathrm{s}}^{2}$, to obtain an EoS that has been reinvented many times $[17,19]$ :

$$
\frac{\alpha_{\mathrm{s}} \pi^{\mathrm{s}}}{k_{\mathrm{B}} T}=\frac{\psi_{\mathrm{s}}}{\left(1-\psi_{\mathrm{s}}\right)^{2}}-\beta \psi_{\mathrm{s}}^{2}
$$

here, $\beta$ is the so-called lateral attraction parameter. The attraction term in Eq (2) is semiempirical; in result, the model is unsatisfactory at high $\psi_{\mathrm{s}}$ and large values of $\beta$ [23]. A more reliable EoS for attractive molecules is offered by the sticky disc (SD) model of Ivanov et al. [20-23]:

$$
\frac{\alpha_{\mathrm{s}} \pi^{\mathrm{s}}}{k_{\mathrm{B}} T}=\frac{R_{\beta}-1}{2 \beta\left(1-\psi_{\mathrm{s}}\right)}, \text { where } \quad R_{\beta}=\sqrt{1+4 \beta \frac{\psi_{\mathrm{s}}}{1-\psi_{\mathrm{s}}}} .
$$

The corresponding SD surface activity coefficient $\gamma_{\mathrm{s}}$ follows from the Gibbs isotherm, $\mathrm{d}\left(\alpha_{\mathrm{s}} \pi^{\mathrm{S}} / k_{\mathrm{B}} T\right)=\psi_{\mathrm{s}} \mathrm{d}\left(\ln \gamma_{\mathrm{s}} \psi_{\mathrm{s}}\right)$, as: 


$$
\ln \gamma_{\mathrm{s}}=-\ln \left(1-\psi_{\mathrm{s}}\right)+\left(2+\frac{1}{\beta}\right) \ln \frac{2}{1+R_{\beta}}+\frac{\psi_{\mathrm{s}}\left(4-3 \psi_{\mathrm{s}}\right)}{\left(1-\psi_{\mathrm{s}}\right)^{2}} \frac{2}{1+R_{\beta}} .
$$

The respective adsorption isotherm (the chemical equilibrium condition for the surfactant at the surface and in the bulk) of the SD model reads

$$
\alpha_{\mathrm{s}} K_{\mathrm{a}} C_{\mathrm{s}}=\gamma_{\mathrm{s}} \psi_{\mathrm{s}}
$$

where $K_{\mathrm{a}}[\mathrm{m}]$ is the adsorption constant $\left(R T \ln K_{\mathrm{a}}\right.$ is the adsorption free energy) and $C_{\mathrm{s}}\left[\mathrm{m}^{-3}\right]$ is the concentration of the surfactant [23].

An important feature of the EoS (2)\&(3) is that they, unlike all other popular adsorption models, agree with the theoretically expected virial expansion [23]:

$$
\frac{\alpha_{\mathrm{s}} \pi^{\mathrm{s}}}{k_{\mathrm{B}} T} \stackrel{\psi_{\mathrm{s}} \rightarrow 0}{\longrightarrow} \psi_{\mathrm{s}}+\frac{B_{2}}{\alpha_{\mathrm{s}}} \psi_{\mathrm{s}}^{2}+\ldots, \text { with } 2^{\text {nd }} \text { virial coefficient } B_{2}=2 \alpha_{\mathrm{s}}-\alpha_{\mathrm{s}} \beta \text {. }
$$

The $2 \alpha_{\mathrm{s}}$ term in Eq. (6) is the repulsive hard-disc part of the surface virial coefficient, and $-\alpha_{\mathrm{s}} \beta$ is its attractive part. For 1 -component liquid made of attracting hard discs, $\beta$ can be computed from the binary attraction potential $u_{\mathrm{attr}}(\rho)$ as

$$
\beta=\frac{1}{R_{\mathrm{s}}^{2}} \int_{2 R_{\mathrm{s}}}^{\infty}\left(\mathrm{e}^{-u_{\text {attr }}(\rho) / k_{\mathrm{B}} T}-1\right) \rho \mathrm{d} \rho,
$$

where $R_{\mathrm{S}}$ is the hard-disc radius of the surfactant $\left(\alpha_{\mathrm{s}}=\pi R_{\mathrm{s}}^{2}\right)$, and $\rho$ is distance between the interacting molecules. A simple expression for $u_{\text {attr }}$ at W|A was proposed in Ref. [19] - two adsorbed surfactant molecules of straight hydrocarbon chains separated by a distance $\rho$ experience van der Waals attraction between each other of potential

$$
u_{\text {attr }}(\rho)=-\frac{n L_{\mathrm{CH}_{2}}}{4 l_{\mathrm{CH}_{2}} \rho^{5}}\left(3 \arctan \frac{n l_{\mathrm{CH}_{2}}}{\rho}+\frac{n l_{\mathrm{CH}_{2}} \rho}{\rho^{2}+n^{2} l_{\mathrm{CH}_{2}}^{2}}\right) ;
$$

here, $n$ is the number of methylene $\left(\mathrm{CH}_{2}\right)$ groups in the hydrocarbon chain assumed to stick above the aqueous phase (those that remain immersed in water should contribute negligibly to the van der Waals attraction), $L_{\mathrm{CH}_{2}}=4.24 \times 10^{-78} \mathrm{~m}^{6} \mathrm{~J}$ is the London constant for the interaction between two $\mathrm{CH}_{2}$ groups, and $l_{\mathrm{CH}_{2}}=1.26 \AA$ is the length of a $\mathrm{CH}_{2}$ group along the hydrophobic chain [25]. The SD model (3)-(5), with $\beta$ computed via Eqs (7)\&(8), has been found to agree very well with surface tension data for many cohesive monolayers at W|A [23]. However, just as HFL, the SD model does not account explicitly for the presence of solvent molecules in the plane of the monolayer, which makes Frumkin's criticism relevant [1], as outlined in the introduction.

\subsection{A hard-disc liquid mixture}

Let the polar head groups of the adsorbed surfactant molecules be located in a 1-molecule thick surface layer containing solvent, as schematized in Figure 1. The justification for this monolayer model has been given by Defay and Prigogine, sec. XI.6 of Ref. [26]: the density drop in a surface layer indeed occurs within 1-2 molecular diameters, unless the system is close to a critical point. The surface layer in the absence of surfactant is known to be of decreased density in comparison with the bulk fluid - let this surface density correspond to a certain surface coverage of $\psi_{\mathrm{w} 0}$ with solvent molecules. The presence of surfactant (covering a fraction $\psi_{\mathrm{s}}$ of the surface) is expected to expulse the solvent from the surface layer, i.e. in the presence of surfactant, the solvent's $\psi_{\mathrm{w}}$ must be smaller than $\psi_{\mathrm{w} 0}$. 


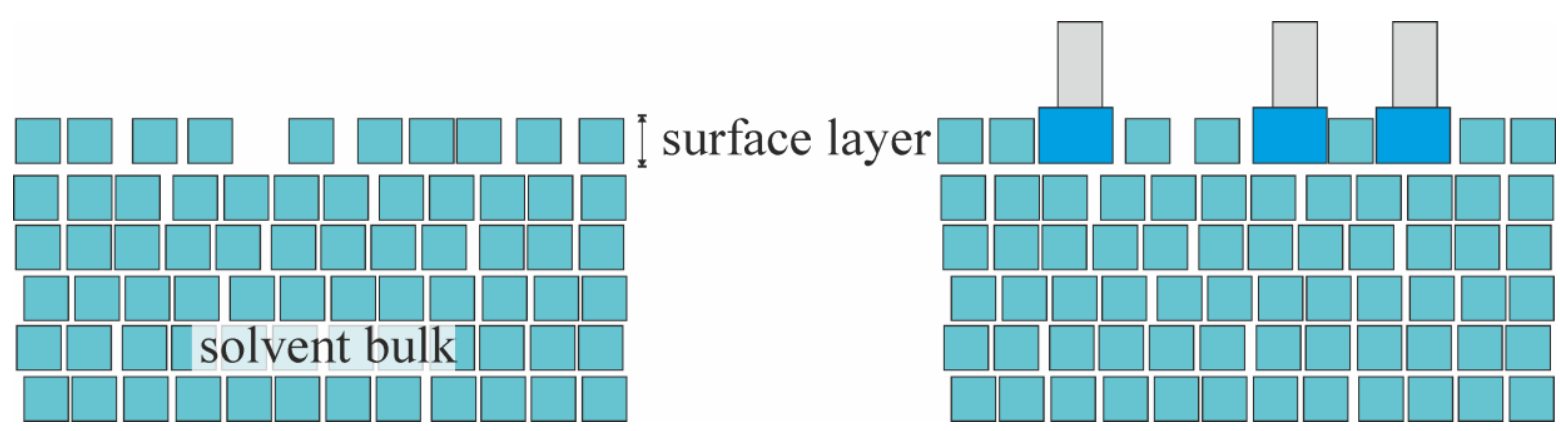

Figure 1. Schematic of the model: structure of the surface layer before and after the adsorption of surfactant.

The surface layer is further assumed to behave as a mixture of hard discs of different areas, thus upgrading HFL model to account for the solvent present at the surface (or alternatively, upgrading Frumkin's formulation of the Langmuir model in Refs. [1,6] to make it delocalized). The EoS predicted by the scaled particle theory for a hard-disc mixture has been derived by Lebowitz, Helfand and Praestgaard (LHP) [27]; for a binary mixture, their eq 6.7 can be written as

$$
\frac{\alpha_{\mathrm{s}} \pi^{\mathrm{s}}}{k_{\mathrm{B}} T}=\frac{\psi_{\mathrm{s}}+r \psi_{\mathrm{w}}-(\sqrt{r}-1)^{2} \psi_{\mathrm{w}} \psi_{\mathrm{s}}}{\left(1-\psi_{\mathrm{s}}-\psi_{\mathrm{w}}\right)^{2}}-\frac{r \psi_{\mathrm{w} 0}}{\left(1-\psi_{\mathrm{w} 0}\right)^{2}} ;
$$

here, $\psi_{\mathrm{w}} \equiv \alpha_{\mathrm{w}} \Gamma_{\mathrm{w}}$ is the surface fraction covered by solvent (water) molecules; $\Gamma_{\mathrm{w}}$ is the surface density of solvent molecules in the plane of the monolayer (its relation to the Gibbs adsorption is discussed in the supplement S1); $\alpha_{\mathrm{w}}$ is the hard-disc area of a solvent molecule; $\psi_{\mathrm{w} 0}=\alpha_{\mathrm{w}} \Gamma_{\mathrm{w} 0}$, where $\Gamma_{\mathrm{w} 0}$ is the surface density of solvent molecules at the neat surface; $r \equiv \alpha_{\mathrm{s}} / \alpha_{\mathrm{w}}$ is the surfactant/solvent hard-disc area ratio. The Gibbs fundamental isotherm relates this EoS to the chemical potentials (or equivalently, to the surface activity coefficients $\gamma_{\mathrm{s}}$ and $\gamma_{\mathrm{w}}$ ) of the two components:

$$
\mathrm{d} \frac{\alpha_{\mathrm{s}} \pi^{\mathrm{s}}}{k_{\mathrm{B}} T}=r \psi_{\mathrm{w}} \mathrm{d}\left(\ln \gamma_{\mathrm{w}} \psi_{\mathrm{w}}\right)+\psi_{\mathrm{s}} \mathrm{d}\left(\ln \gamma_{\mathrm{s}} \psi_{\mathrm{s}}\right)
$$

The position of the Gibbs equimolecular surface that corresponds to this model is analysed in the supplement S1. For a binary mixture, both $\gamma_{\mathrm{s}}$ and $\gamma_{\mathrm{w}}$ can be derived from Eq (10). The derivation is trivial but lengthy and we shall skip it for clarity (a schematic of the derivation is given in S5). The final result reads:

$$
\begin{aligned}
& \ln \gamma_{\mathrm{w}}=-\ln \left(1-\psi_{\mathrm{s}}-\psi_{\mathrm{w}}\right)+\frac{\psi_{\mathrm{w}}\left(3-2 \psi_{\mathrm{w}}\right)+\psi_{\mathrm{s}}\left[\frac{2}{\sqrt{r}}\left(1-\psi_{\mathrm{s}}\right)+\frac{1-\psi_{\mathrm{w}}}{r}-3 \psi_{\mathrm{w}}\right]}{\left(1-\psi_{\mathrm{s}}-\psi_{\mathrm{w}}\right)^{2}} \\
& \ln \gamma_{\mathrm{s}}=-\ln \left(1-\psi_{\mathrm{s}}-\psi_{\mathrm{w}}\right)+\frac{\psi_{\mathrm{s}}\left(3-2 \psi_{\mathrm{s}}\right)+\psi_{\mathrm{w}}\left[2 \sqrt{r}\left(1-\psi_{\mathrm{w}}\right)+r\left(1-\psi_{\mathrm{s}}\right)-3 \psi_{\mathrm{s}}\right]}{\left(1-\psi_{\mathrm{s}}-\psi_{\mathrm{w}}\right)^{2}} .
\end{aligned}
$$

These activity coefficients correspond to a standard state of infinitely dilute monolayer, i.e. $\gamma_{\mathrm{w}}=\gamma_{\mathrm{s}}=1$ when $\psi_{\mathrm{w}}=\psi_{\mathrm{s}}=0$; this is in contrast to Eq (4), which involves the implicit assumption that $\gamma_{\mathrm{s}}=1$ when $\psi_{\mathrm{s}}=0$ and $\psi_{\mathrm{w}}=\psi_{\mathrm{w} 0}-$ i.e. the standard state there is the neat surface. The new standard state requires a different general form of the adsorption isotherm for the surfactant - instead of Eq (5), one has to use

$$
\alpha_{\mathrm{s}} K_{\mathrm{a}} C_{\mathrm{s}}=\gamma_{\mathrm{s}} \psi_{\mathrm{s}} / \gamma_{\mathrm{s} 0}
$$

where $\gamma_{\mathrm{s} 0}=\gamma_{\mathrm{s}}\left(\psi_{\mathrm{s}}=0, \psi_{\mathrm{w}}=\psi_{\mathrm{w} 0}\right)$ is the activity of the surfactant at the neat surface in a monolayer dilute with respect to the surfactant only (but not with respect to the solvent). 
The amount of water $\psi_{\mathrm{w}}$ in the surface layer decreases with the increase of the adsorption of surfactant. However, if the bulk surfactant solution is dilute, then, to a good approximation, water is of constant chemical potential independent of the amount of dissolved surfactant (a general approach to the case of concentrated solutions is considered briefly in S4). The dependence of $\psi_{\mathrm{w}}$ on $\psi_{\mathrm{s}}$ at constant chemical potential of the solvent follows from the equilibrium condition $\gamma_{\mathrm{w}} \psi_{\mathrm{w}}=\gamma_{\mathrm{w} 0} \psi_{\mathrm{w} 0}$ (i.e. the activity of the solvent in the monolayer is equal to that of solvent at the neat surface as both are equal to the bulk activity of the solvent); here, the subscript 0 indicates again neat water surface $\left(\gamma_{\mathrm{w} 0}\right.$ is $\gamma_{\mathrm{w}}$ at $\left.\psi_{\mathrm{s}}=0\right)$. This condition, together with the first $\mathrm{Eq}(11)$, leads to the adsorption isotherm of the solvent:

$$
\frac{\psi_{\mathrm{w}}}{1-\psi_{\mathrm{s}}-\psi_{\mathrm{w}}} \mathrm{e}^{\frac{\psi_{\mathrm{w}}\left(3-2 \psi_{\mathrm{w}}\right)+\psi_{\mathrm{s}}\left[\frac{2}{\sqrt{r}}\left(1-\psi_{\mathrm{s}}\right)+\frac{1-\psi_{\mathrm{w}}}{r}-3 \psi_{\mathrm{w}}\right]}{\left(1-\psi_{\mathrm{s}}-\psi_{\mathrm{w}}\right)^{2}}}=\frac{\psi_{\mathrm{w} 0}}{1-\psi_{\mathrm{w} 0}} \mathrm{e}^{\frac{\psi_{\mathrm{w} 0}\left(3-2 \psi_{\mathrm{w}}\right)}{\left(1-\psi_{\mathrm{w} 0}\right)^{2}}} .
$$

The adsorption isotherm of the surfactant is obtained by substituting the surface activity coefficient $\gamma_{\mathrm{s}}$, Eq (11), into Eq (12):

$$
\alpha_{\mathrm{s}} K_{\mathrm{a}} C_{\mathrm{s}}=\frac{\psi_{\mathrm{s}}\left(1-\psi_{\mathrm{w} 0}\right)}{1-\psi_{\mathrm{s}}-\psi_{\mathrm{w}}} \mathrm{e}^{\frac{\psi_{\mathrm{s}}\left(3-2 \psi_{\mathrm{s}}\right)+\psi_{\mathrm{w}}\left[2 \sqrt{r}\left(1-\psi_{\mathrm{w}}\right)+r\left(1-\psi_{\mathrm{s}}\right)-3 \psi_{\mathrm{s}}\right]}{\left(1-\psi_{\mathrm{s}}-\psi_{\mathrm{w}}\right)^{2}}-\frac{\psi_{\mathrm{w} 0}\left[2 \sqrt{r}\left(1-\psi_{\mathrm{w} 0}\right)+r\right]}{\left(1-\psi_{\mathrm{w} 0}\right)^{2}}} .
$$

The two adsorption isotherms (13)\&(14), together with the LHP EoS (9), completely define the state of the surface (i.e. $\pi^{\mathrm{S}}, \psi_{\mathrm{w}}$, and $\psi_{\mathrm{s}}$ are defined as functions of $C_{\mathrm{s}}$ ), if the hard-disc areas $\alpha_{\mathrm{s}}$ of the surfactant and $\alpha_{\mathrm{w}}$ of the solvent, and the neat surface density $\psi_{\mathrm{w} 0}$ are known. In the limit of dry monolayer $\left(\psi_{\mathrm{w}} \rightarrow 0\right)$, the isotherm of the surfactant simplifies to the one following from the HFL model, cf. S3.

The van der Waals radius of a water molecule is $1.39 \AA$, which corresponds to hard-disc area of $\alpha_{\mathrm{w}}=6.07 \AA^{2}$ and hard-sphere volume $v_{\mathrm{w}}=11.25 \AA^{3}$. The molecular volume (molar volume divided by Avogadro's number) of pure water at $25{ }^{\circ} \mathrm{C}$ is $V_{\mathrm{w}}=30.01 \AA^{3}$. Assuming that the density of the surface layer is similar to that of the bulk, one can estimate the molecular area of the neat surface at $A_{\mathrm{w}}=V_{\mathrm{w}}^{2 / 3}=9.66 \AA^{2}$, which corresponds to a coverage of $\psi_{\mathrm{w} 0}=\alpha_{\mathrm{w}} / A_{\mathrm{w}}$ $=0.63$ of the neat surface. However, the surface layer is well-known to be of decreased density (e.g., sec. XI.6 of Ref. [26]). A measure of this decrease is the so-called "hydrophobic gap" width: the X-ray reflectivity of the interface between water and a hydrophobic material has been shown to be the same as if a vacuum gap of thickness $1.4 \AA$ [28] existed between two bulk phases, aqueous and hydrophobic, of normal density. This shows that the density of the surface layer of water (that is $\sim 2.8 \AA$ thick) is about half the bulk density; hence, the density of the surface layer is about half the above estimate, i.e. $\psi_{\mathrm{w} 0}=0.62 / 2=0.31$, provided that the distance between the "layers" does not increase near the interface. However, the latter distance most probably increases (see S1); therefore, the actual value of $\psi_{\mathrm{w} 0}$ can be expected to fall in the range $0.31-0.63$. It is noteworthy that $\psi_{\mathrm{w} 0}$, a characteristic of the neat surface, should be independent of the nature of the surfactant forming the monolayer.

The dependence of $\psi_{\mathrm{w}}$ on $\psi_{\mathrm{s}}$ that follows from Eq (13), with $\alpha_{\mathrm{w}}=6.07 \AA^{2}$ and $\alpha_{\mathrm{s}}=16.5$ $\AA^{2}$ (as for a fatty alcohol [22]), is illustrated in Figure 2 for three values of $\psi_{\mathrm{w} 0}$. It is seen that this dependence is linear at $\psi_{\mathrm{s}} \rightarrow 0$. An expansion in series of Eq (13) gives for this linear region the expression:

$$
\psi_{\mathrm{w}}=\psi_{\mathrm{w} 0}-\frac{2 \sqrt{r}\left(1-\psi_{\mathrm{w} 0}\right)+\left(1-\psi_{\mathrm{w} 0}\right)^{2}+r\left(1+\psi_{\mathrm{w} 0}\right)}{r\left(1+\psi_{\mathrm{w} 0}\right)} \psi_{\mathrm{w} 0} \psi_{\mathrm{s}}+\mathrm{O}\left(\psi_{\mathrm{s}}^{2}\right) .
$$

As the density of the surfactant monolayer increases, a point is reached $\left(\psi_{\mathrm{s}}=0.7-0.8\right)$ where the solvent is nearly completely expulsed from the surface layer. Above this point, $\psi_{\mathrm{w}}$ decreases 
exponentially with $\psi_{\mathrm{s}}$; this case (corresponding to series with respect to small $\psi_{\mathrm{w}}$ ) is analysed in S3.

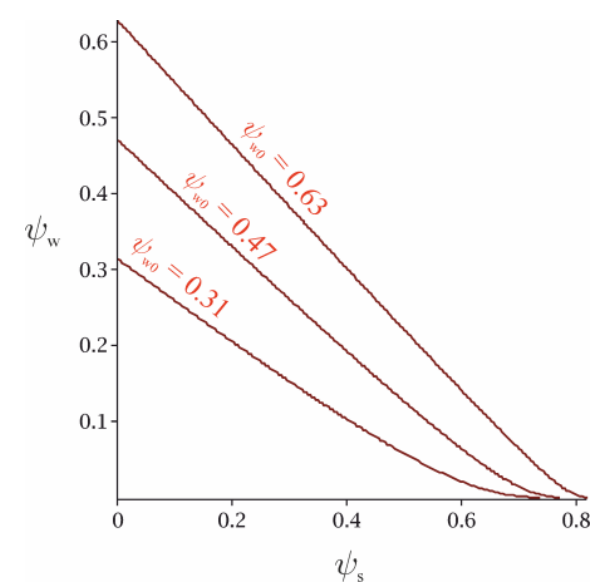

Figure 2. Expulsion of the solvent from the adsorption monolayer: surface coverage with solvent molecules, $\psi_{\mathrm{w}}=\alpha_{\mathrm{w}} \Gamma_{\mathrm{w}}$, as a function of the surfactant area fraction, $\psi_{\mathrm{s}}=\alpha_{\mathrm{s}} \Gamma_{\mathrm{s}}$, according to Eq (13) of the hard-disc mixture model $\left(\alpha_{\mathrm{w}}=6.07 \AA^{2}, \alpha_{\mathrm{s}}=16.5 \AA^{2}\right.$, and 3 different values of $\left.\psi_{\mathrm{w} 0} ; 25^{\circ} \mathrm{C}\right)$.

By substitution of Eq (15) into the LHP EoS (9) and expansion in series with respect to $\psi$ s (an approximation corresponding to a dilute 2D solution of surfactant), one obtains the following 2D osmotic virial expansion [29] of the EoS of the monolayer at fixed chemical potential of the solvent:

$$
\frac{\alpha_{\mathrm{s}} \pi^{\mathrm{s}}}{k_{\mathrm{B}} T}=\psi_{\mathrm{s}}+\left(2-\beta_{\text {osm }}\right) \psi_{\mathrm{s}}^{2}+\ldots, \text { where } \quad \beta_{\text {osm }}=\frac{2 r+4 \sqrt{r}+1-\psi_{\mathrm{w} 0}}{2 r\left(1+\psi_{\mathrm{w} 0}\right)} \psi_{\mathrm{w} 0} .
$$

The comparison of the osmotic virial expansion (16) to the 1-component 2D gas virial expansion (6) will show that the osmotic effect on the EoS of a hard-disc monolayer is equivalent to an effective lateral attraction and leads to increased cohesiveness of the monolayer. This is illustrated in Figure 3, where the hard-disc mixture model (the LHP EoS (9) solved numerically together with the solvent's adsorption isotherm (13) for $\pi^{\mathrm{S}}\left(\psi_{\mathrm{s}}\right)$ and $\left.\psi_{\mathrm{w}}\right)$ is compared to the $\pi^{\mathrm{S}}\left(\psi_{\mathrm{s}}\right)$ dependence of a 1-component hard-disc monolayer according to the HFL EoS (1), which is the limit of LHP theory in the absence of solvent. Evidently, the solvent has a significant effect on the properties of the monolayer: in the whole range of coverages, LHP predicts lower surface pressure than HFL, which corresponds to effective cohesion between the adsorbed molecules. According to $\mathrm{Eq}$ (16), the value of $\beta$ corresponding to this "cohesion" is a function of the density $\psi_{\mathrm{w} 0}$ of the surface layer at the neat surface and the surfactant/solvent hard-disc area ratio $r$. The osmotic attraction is larger for smaller surfactant

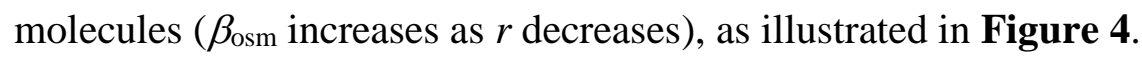

As we shall see in the following sections, the introduction of effective osmotic attraction is useful as it allows the EoS of a cohesive surfactant monolayer in the presence of solvent to be simplified considerably. However, we should stress that the osmotic "attraction" does not correspond to a real interaction between the adsorbed surfactant molecules. The physical explanation of the solvent-induced cohesion is easy to understand if one considers the definition of the surface pressure as a surface excess of the osmotic pressure (e.g., [18]). The penetration of water into the monolayer corresponds to dilution of the surface layer, so it decreases the osmotic pressure in this 2D solution of surfactant in water. Hence, the Gibbs excess of the osmotic pressure - the surface pressure $\pi^{S}$ - also decreases. The drop of $\pi^{S}$ corresponds to a more cohesive monolayer (according to the definition of cohesiveness as negative deviations 
from the ideal gas EoS). Thus, the effect of the solvent penetration in the surface layer appears as osmotic cohesion of the surfactant monolayer.

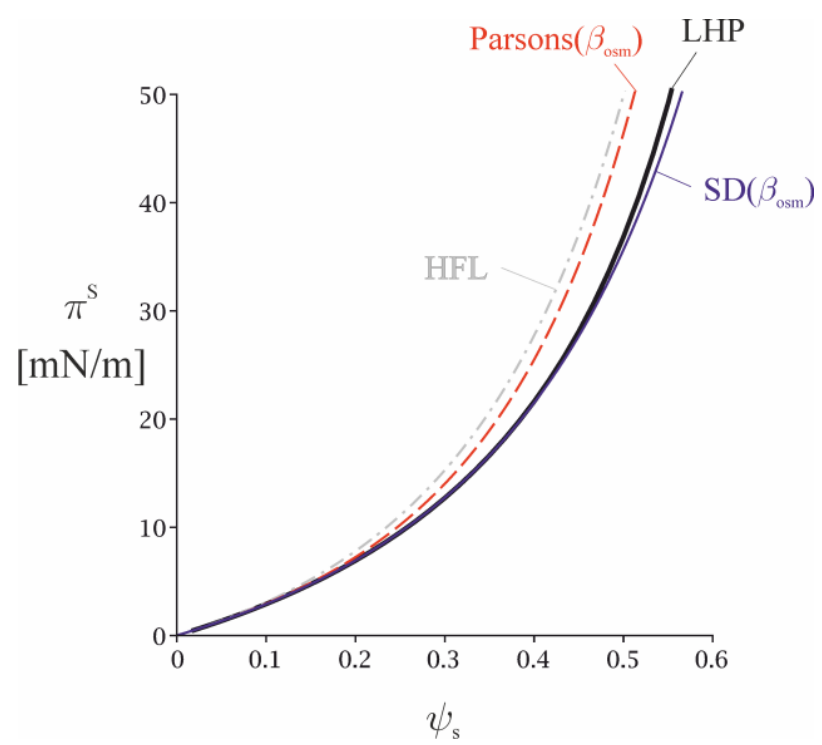

Figure 3. Surface pressure $\pi^{\mathrm{S}}$ vs. surfactant coverage $\psi_{\mathrm{s}}=\alpha_{\mathrm{s}} \Gamma_{\mathrm{s}}$, according to the LHP EoS (9) and the isotherm (13) for 2-component hard-disc fluid, accounting explicitly for the osmotic effect (black solid line, $\alpha_{\mathrm{s}}=16.5 \AA^{2}, \psi_{\mathrm{w} 0}=0.31,25{ }^{\circ} \mathrm{C}$ ). It is compared to the HFL model which neglects the solvent in the surface layer (grey dash-dot, Eq (1)). The osmotic effect can be modelled as an effective attraction by setting the lateral attraction parameter $\beta$ of the EoS

(2)\&(3) of Parsons (red dash) and SD (blue solid) equal to the effective osmotic attraction $\beta_{\text {osm, }}$ Eq (16). The 1-component sticky disc model approximates well the exact 2-component LHP result.

Our results can be compared with the simplistic model of Chattoraj and Birdi, who have generalized Volmer's EoS to include the hard-disc area of the solvent (cf. their eq 5.15 [18]):

$$
\frac{\alpha_{\mathrm{s}} \pi^{\mathrm{s}}}{k_{\mathrm{B}} T}=\frac{\psi_{\mathrm{s}}}{1-(1-1 / r) \psi_{\mathrm{s}}} ;
$$

similar equation has been obtained by Lucassen-Reynders and van den Tempel [30]. For small solvent molecules $(1 / r=0)$, this EoS reduces to Volmer's. The virial expansion of Eq (17) is:

$$
\frac{\alpha_{\mathrm{s}} \pi^{\mathrm{s}}}{k_{\mathrm{B}} T}=\psi_{\mathrm{s}}+(1-1 / r) \psi_{\mathrm{s}}^{2}+\ldots
$$

The cohesive variant of Volmer's EoS is the 2D van der Waals EoS [31,32], $\alpha_{\mathrm{s}} \pi^{\mathrm{S}} / k_{\mathrm{B}} T=\psi_{\mathrm{s}} /\left(1-\psi_{\mathrm{s}}\right)-\beta \psi_{\mathrm{s}}^{2}$ (just as Parsons' models is the cohesive variant of HFL, Eqs (1) $\&(2))$. The virial expansion of the van der Waals EoS reads $\alpha_{\mathrm{s}} \pi^{\mathrm{s}} / k_{\mathrm{B}} T=\psi_{\mathrm{s}}+(1-\beta) \psi_{\mathrm{s}}^{2} \ldots$ Comparison of this expansion with Eq (18) shows that the osmotic attraction following from the model of Chattoraj and Birdi corresponds to $\beta_{\text {osm }}=1 / r$. This result is compared to Eq (16) for $\beta_{\text {osm }}$ of the hard-disc mixture in Figure 4: as seen, the qualitative behavior is similar - the osmotic cohesion is stronger for surfactants of smaller cross-section. However, the model of Chattoraj and Birdi has all problems inherent to Volmer's EoS (discussed in Ref. [23]), e.g., the value of the parameter $(1-1 / r) \alpha_{\mathrm{s}}$ in Eq (17) has, in theory, to be smaller than $\alpha_{\mathrm{s}}$; instead, the fit of experimental tensiometric data with Eq (17) always gives a value of $(1-1 / r) \alpha_{\text {s }}$ larger than the hard-disc area of the surfactant. 


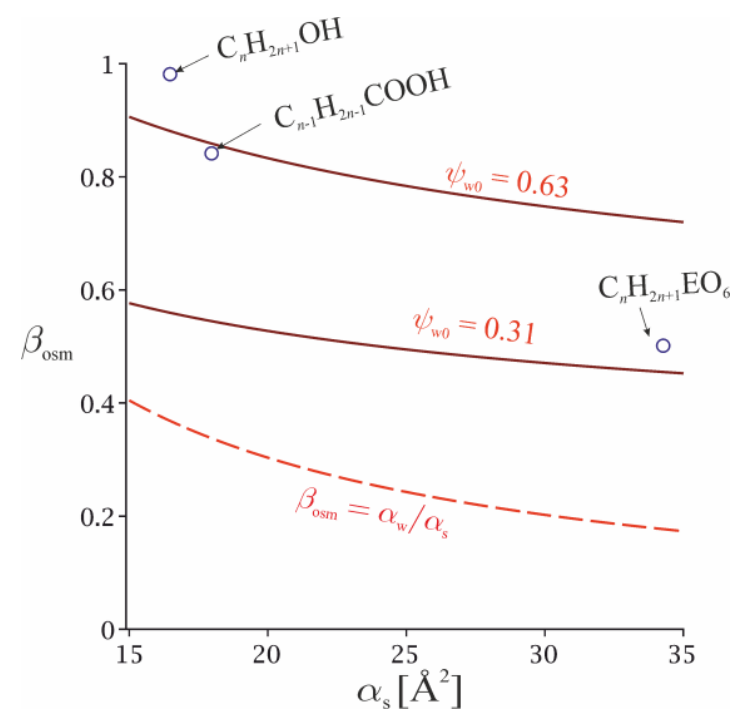

Figure 4. Comparison between Eq (16) for the osmotic attraction parameter $\beta_{\text {osm }}$ vs. the harddisc area of the surfactant $\alpha_{\mathrm{s}}$ (solid lines) and the osmotic lateral attraction parameters obtained from the fit of the tensiometric data for the three homologous series of surfactants in Table 1 (points). The osmotic attraction parameter $\beta_{\mathrm{osm}}=1 / r$ following from the model (17) of Chattoraj and Birdi is given for comparison (dashed line).

\subsection{Modifying 1-component models for cohesive monolayers to account for the osmotic effect}

We have shown in the previous section that the presence of solvent molecules of non-zero crosssection area in the monolayer results in effective cohesion between the surfactants. It can be then expected that a model of a cohesive monolayer, such as Parsons' or the SD EoS, can offer a good approximation to the behaviour of the hard-disc mixture, if their lateral attraction parameter $\beta$ is set equal to $\beta_{\text {osm }}$ from Eq (16). A comparison between the exact LHP model of the mixture (a numerical solution to Eqs (9)\&(13)) with Parsons' EoS (2) and the SD EoS (3) with $\beta=\beta_{\text {osm }}$ is made in Figure 3. It shows that Parsons' model deviates from the exact EoS, while the SD theory indeed approximates LHP adequately - the match is good for any physically reasonable value of $\alpha_{\mathrm{s}}$ when $\psi_{\mathrm{w} 0}=0.31$; however, if $\psi_{\mathrm{w} 0}$ is $>0.5$, the differences become non-negligible. Thus, if it is ensured that the SD model has the correct $2^{\text {nd }}$ virial coefficient including the osmotic effect, this 1-component model becomes nearly equivalent to the more advanced hard-disc mixture theory of LHP.

This finding allows us to make an approximate but straightforward extension of the harddisc mixture model to attractive molecules. A monolayer made of surfactant molecules interacting between each other with an attractive potential $u_{\text {attr, }}$ and containing solvent molecules penetrating the monolayer as sketched in Figure 1, can be expected to follow the 1component SD model (3)-(5), but with lateral attraction parameter given by the sum

$$
\beta=\beta_{\text {attr }}+\beta_{\text {osm }},
$$

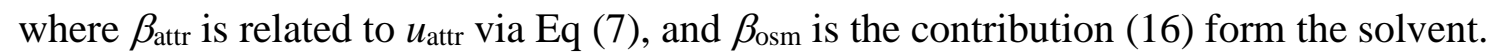

This simple rule explains the apparent success of the SD model in predicting the behaviour of monolayers adsorbed at liquid interfaces found in Ref. [23], despite the fact it neglects the solvent. A more detailed analysis would show that the good coincidence with the experimental data is, in fact, due to a compensation of effects. The formula (7)-(8) for the van der Waals contribution to $\beta$ has been used in Ref. [23] under the assumption that all $\mathrm{CH}_{2}$ groups contribute equally to the lateral attraction $\left(\beta=\beta_{\mathrm{vdW}, n}\right)$. However, there is enough evidence that one 
methylene group remains immersed in the aqueous phase, probably due to the polarization effect of the polar head group on the adjacent $\mathrm{CH}_{2}$. The immersed $\mathrm{CH}_{2}$ should not contribute significantly to $\beta$, as the van der Waals interaction between $\mathrm{CH}_{2}$ groups through water is weak [25]. This immersion effect is especially clear (i) with fatty acids, where only $n-1$ carbon atoms should contribute to $\beta$ as the first carbon is in a carbonyl $(>\mathrm{C}=\mathrm{O})$ group (whereas in Ref. [23] it was assumed that all $n$ carbons contribute); and (ii) with alkyldymethylphosphineoxides, where the two $\mathrm{CH}_{3}$ groups attached to the polar head group contribute neither to the adsorption constant $K_{\mathrm{a}}$ nor to the attraction parameter $\beta$ of these surfactants, i.e. these methyl groups behave as a part of the polar head [23]. A similar immersion effect has been noticed with micelles (Chap. 3 and Eq. 6-4 of Tanford [33]; see also Ref. [34]). Thus, instead of our previous assumption $\beta=\beta_{\mathrm{vdW}, n}$, a more realistic model of $\beta$ would account explicitly for both the solvent molecules in the monolayer and the immersion of one $\mathrm{CH}_{2}$ group as:

$$
\beta=\beta_{\mathrm{vdW}, n-1}+\beta_{\text {osm }} \text {. }
$$

A hydrophobic chain shorter by one methylene group corresponds to a smaller van der Waals attraction, which compensates for the neglected osmotic attraction (i.e. $\beta_{\mathrm{vdW}, n-1}+\beta_{\mathrm{osm}} \approx \beta_{\mathrm{vdW}, n \text { ) }}$ and leads ultimately to the adequate comparison with the experiment observed in Ref. [23]. In the following section, we will take an explicit account for both the osmotic and the immersion effects when comparing with tensiometric data, to show that the compensation is often, but not always, efficient.

Before turning to experimental data, let us summarize the limitations of the sticky disc model:

(i) Water is an associated liquid. The hard-sphere models are quite successful in predicting numerous properties of aqueous solutions [35], yet, a hard-disc model can only offer a first approximation to the aqueous surface. Corrections for the association and the dipole-dipole interactions are an important next step towards a thorough theory (the SD model involves only surfactant-surfactant interaction).

(ii) The monolayer assumption is rather crude.

(iii) The SD model corrected for the osmotic effect does not coincide completely with the 2-component LHP. The assumption (20) for additivity of the van der Waals attraction and the osmotic cohesion is not necessary accurate.

(iv) The model of the lateral attraction is also crude, as it neglects, e.g., the direct interaction between the polar head groups. The role of the normal dipole moment of the monolayer is also neglected [36].

\section{Comparison with experimental data}

The most common experimental source of information for the state of soluble monolayers is tensiometry ( $\sigma$ vs. $C_{\mathrm{s}}$ data). In order to determine the experimental value of the osmotic attraction parameter $\beta_{\text {osm }}$, we will use surface tension data at $\mathrm{W} \mid \mathrm{A}$ for three homologous series of surfactants having head-groups of different hard-disc areas: alkanols, non-dissociated carboxylic acids, and hexaethyleneglycol alkyl ethers (alkanol hexaethoxylates, $\mathrm{C}_{n} \mathrm{H}_{2 n+1} \mathrm{EO}_{6}$ ). A direct fit of tensiometric data for a single surfactant with any adsorption model suffers from large uncertainty of the involved adsorption parameters, especially $\alpha_{\mathrm{s}}$ and $\beta$ [23]. In addition, the disagreement between the available data for the same surfactant from different authors often leads to misleading results (an example is given below). To minimize these errors, we will use the theoretically expected values for all adsorption parameters which can be determined independently, drawing on the works of Ivanov et al. [19,21,22,23,37], as follows. 
(i) We use crystallographic or monolayer collapse data (cf. S1) to determine the hard-disc area $\alpha_{\mathrm{s}}$ of the surfactant independently, instead of fitting it. The alcohol group is of cross-section area smaller than that of the hydrocarbon chain, so we used crystallographic data for solid alkanes [38,39] together with area of collapse of insoluble monolayers of alcohols $[40,41]$ : these data yield an average area per molecule in a close-packed structure of $\alpha_{\perp}=18.2 \pm 0.4 \AA^{2}$. This value must be corrected by a packing factor: for hexagonal packing, $\alpha_{\perp}$ must be divided by 1.10 (the ratio between the area of a hexagon and the inscribed circle) to obtain the actual area $\alpha_{\mathrm{s}}=16.5 \pm 0.4 \AA^{2}$ of the hard disc. For acids, we used data for the collapse area of several insoluble long-chained homologues $[17,42,43,44]$. The average value of these data is $\alpha_{\perp}=$ $19.8 \pm 0.8 \AA^{2}$, which is close to the crystallographic area $20.5 \AA^{2}$ quoted by Langmuir [42], and to the average $\alpha_{\perp}=20.05 \AA^{2}$ following from the crystallographic data of Bond [45] (calculated as explained in $\mathrm{S} 1$ ). The relatively large uncertainty $\left( \pm 0.8 \AA^{2}\right)$ is probably related to the slightly different $\alpha_{\perp}$ of the acids with even or odd number of carbons (from Bond's data, $\alpha_{\perp}=19.0$ or $20.4 \AA^{2}$, respectively [45]). Using the correction factor for close packing 1.10 , we find for the hard-disc area $\alpha_{\mathrm{s}}=18.0 \pm 0.8 \AA^{2}$ for acids. The collapse area of the ethoxylate monolayer can be estimated from the surface pressure isotherm of Lange and Jeschke [46] for hexaethoxylate spread on concentrated $\mathrm{NaNO}_{3}$, which ends at $\alpha_{\perp}=37.8 \AA^{2}$, where presumably the collapse occurred; with the correction factor 1.1 for hexagonal packing, this gives $\alpha_{\mathrm{s}}=34.2 \AA^{2}$. However, the collapse area has, in principle, a small but non-negligible dependence on the salt concentration (as evident, e.g., from fig. 5 in Ref. [41]), so instead of using $\alpha_{\mathrm{s}}=34.2 \AA^{2}$, we left $\alpha_{\mathrm{s}}$ of $\mathrm{C}_{n} \mathrm{H}_{2 n+1} \mathrm{EO}_{6}$ as an adjustable parameter to be determined from the experimental data. The areas $\alpha_{\perp}$ and $\alpha_{\mathrm{s}}$ are, to a good approximation, independent of $n$.

(ii) For the dependence of the adsorption constant $K_{\mathrm{a}}$ on $n$, we utilize Traube's rule:

$$
\ln K_{\mathrm{a}}=\ln K_{\mathrm{a} 0}+n \Delta \mu_{\mathrm{CH}_{2}} / k_{\mathrm{B}} T .
$$

For all surfactants, we used the known value for the free energy of transfer of a $\mathrm{CH}_{2}$ from air to water, $\Delta \mu_{\mathrm{CH}_{2}} / k_{\mathrm{B}} T=1.04 \pm 0.06$ [37], an average obtained from adsorption data for numerous ionic and non-ionic surfactants. The intercept $\ln K_{\mathrm{a} 0}$ in $\mathrm{Eq}(21)$ is a characteristic of the whole homologous series. Since it is a very sensitive parameter, it will be left as a fitting parameter. To check how reasonable are the obtained values of $\ln K_{\mathrm{a} 0}$, we will use the recent theory of Ivanov et al., which can be formulated as:

$$
\ln K_{\mathrm{a} 0}=\ln \delta_{\mathrm{a}}+\alpha_{\perp} \sigma_{0} / k_{\mathrm{B}} T ;
$$

this is eq. 27 in Ref. [23], with contribution of the polar head $\Delta \mu_{\text {head }}=-\Delta \mu_{\mathrm{CH}_{2}}$ due to the immersion of the methylene group adjacent to the head group, and $\Delta \mu_{\mathrm{CH}_{3}} \approx 2 \Delta \mu_{\mathrm{CH}_{2}}$ for the energy of transfer of a $\mathrm{CH}_{3}$ group from air to water. Here, $\delta_{\mathrm{a}}=l_{\mathrm{CH}_{2}} k_{\mathrm{B}} T / 2 \Delta \mu_{\mathrm{CH}_{2}}$ is Ivanov's adsorption length; its value is very different from the empirical assumption that $\delta_{\mathrm{a}}=n l_{\mathrm{CH}_{2}}$ which is very common in the literature [47,48]. The term $\alpha_{\perp} \sigma_{0}$ stands for the free energy of the portion of neat surface that disappears upon adsorption of a molecule, and has occurred in several mechanistic models of $K_{\mathrm{a}}$ (e.g., [49]). The term $\alpha \perp \sigma_{0}$ has been shown to have a large contribution to the heat of adsorption [37]; it also explains the effect of the nature of the oil phase on the adsorption constant at various water|oil interfaces [22]. In this work, we will test another prediction: according to $\mathrm{Eq}(22)$, the energy $\alpha_{\perp} \sigma_{0}$ is the sole reason for the different adsorption constants of the three surfactant series at the same value of $n$.

(iii) Finally, for the lateral attraction parameter, we use Eqs (7), (8)\&(20), with $\beta_{\mathrm{vdW}, n-1}$ corresponding to attractive potential $u_{\text {attr }}$ involving only those $n-1 \mathrm{CH}_{2}$ groups that are not immersed in the aqueous phase. Written explicitly in a form convenient for numerical integration, $\mathrm{Eq}(20)$ reads: 


$$
\begin{gathered}
\beta=\beta_{\mathrm{osm}}+\frac{\pi}{\tilde{\alpha}_{\mathrm{s}}} \int_{2 \sqrt{\tilde{s}_{\mathrm{s}} / \pi}}^{\infty}\left[\exp \left(\frac{\frac{\tilde{\rho}}{\tilde{\rho}^{2}+1}+3 \arctan \frac{1}{\tilde{\rho}}}{4 \tilde{T} \tilde{\rho}^{5}}\right)-1\right] \tilde{\rho} \mathrm{d} \tilde{\rho} \text {, where } \\
\tilde{T}=(n-1)^{4} l_{\mathrm{CH}_{2}}^{6} k_{\mathrm{B}} T / L_{\mathrm{CH}_{2}}, \quad \tilde{\alpha}_{\mathrm{s}}=\alpha_{\mathrm{s}} /(n-1)^{2} l_{\mathrm{CH}_{2}}^{2} .
\end{gathered}
$$

To our knowledge, Eqs (8)\&(23) are the only published statistical mechanical model for $\beta$ (apart from the semi-empirical linear formula of Smith [17]). We will leave the osmotic contribution $\beta_{\text {osm }}$ as a free fitting parameter, assuming that it is independent of $n$, as predicted by the hard-disc mixture formula (16).

Let us note that we have previously shown that the hard-disc area $\alpha_{\mathrm{s}}$ can be substituted with what follows from collapse and crystallographic data only if an appropriate delocalized model is used - the areas of the molecule following from the popular models of Volmer, van der Waals, Langmuir, Frumkin and others are always no more than empirical parameters much higher than the crystallographic area, and are not transferable to, e.g., water|oil interface [23]. In addition, the formulae (7)-(8) for $\beta$ are compatible with the sticky disc model (3), but not with cohesive models based on the correction $\beta \psi_{\mathrm{s}}^{2}$ in the EoS (such as Parsons', van der Waals and Frumkin's) [23].

To recapitulate, instead of using 3 adjustable parameters $\left(K_{\mathrm{a}}, \alpha_{\mathrm{s}}\right.$ and $\beta$ ) for each surfactant in a homologous series (or a total of 21 parameters for 7 alcohols or acids), the thermodynamic models (i-iii) allow us to use two or three parameters $\left(K_{\mathrm{a} 0}, \beta_{\mathrm{osm}}\right.$, and for the $\mathrm{C}_{n} \mathrm{H}_{2 n+1} \mathrm{EO}_{6}-$ also $\alpha_{\mathrm{s}}$ ) for a whole homologous series. Even for these three parameters, we have reliable independent estimates that would let us to judge how reasonable the fitted values are.

The tensiometric dataset used for the optimization for a given homologous series is composed of 3 columns - the dependent experimental variable $\pi^{S}$ measured as a function of the surfactant concentration $C_{\mathrm{s}}$ and the chain length $n$. The respective merit function we use for the optimization has the form:

$$
\operatorname{dev}^{2}\left(K_{\mathrm{a} 0}, \beta_{\mathrm{osm}}\right)=\frac{\sum\left[\pi_{n, i}^{\mathrm{s}}-\pi_{\mathrm{th}}^{\mathrm{s}}\left(C_{i}, n ; K_{\mathrm{a} 0}, \beta_{\mathrm{osm}}\right)\right]^{2}}{N-2},
$$

where $\pi_{n, i}^{\mathrm{S}}$ is the $i^{\text {th }}$ experimental surface pressure value for the $n^{\text {th }}$ homologue, $\pi_{\mathrm{th}}^{\mathrm{S}}$ is the theoretical value following from the SD model (3)-(5) at the $i^{\text {th }}$ experimental surfactant

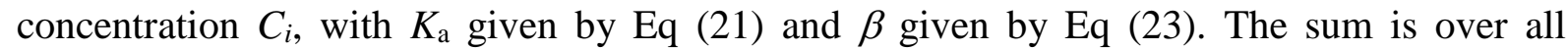
homologues and data points, a total of $N$ points. For the hexaethyleneglycol alkyl ethers, the hard-disc area $\alpha_{\mathrm{s}}$ of the surfactant was also left as a free fitting parameter, while for alcohols and acids we used the areas 16.5 and $18.0 \AA^{2}$ that follow from crystallographic and collapse data.

For alcohols, we used W|A surface tension data for homologues from propanol to decanol from a number of authors [9,50-59], a total of $N=203$ data points; these measurements were done at an average temperature of $21 \pm 1{ }^{\circ} \mathrm{C}$. The data for propanol were corrected for nonideality as explained in Ref. [23]. For the carboxylic acids from $\mathrm{C}_{2} \mathrm{H}_{5} \mathrm{COOH}$ to $\mathrm{C}_{9} \mathrm{H}_{19} \mathrm{COOH}$, we assembled tensiometric data at low $\mathrm{pH}$ and average temperature of $21 \pm 1{ }^{\circ} \mathrm{C}$ from Refs. [10,60-64], $N=163$ points. The data for hexaethyleneglycol monododecyl and monotetradecyl ethers (dodecanol- and tetradecanol hexaethoxylate, $\mathrm{C}_{n} \mathrm{H}_{2 n+1} \mathrm{EO}_{6}$ ) are from Refs. $[9,11,12,65]$, at $25{ }^{\circ} \mathrm{C}, 46$ points. An Excel spreadsheet with the data is provided as a supplement S6.

The results from the minimization of the dispersion (24) are summarized in Table 1 for the three series. There, they are compared with the previous variant of the SD model used in Ref. [23], which neglects the osmotic attraction and the immersion (i.e. $\beta=\beta_{\mathrm{vdW}, n}$ ). The improvement of the standard deviation when the osmotic and the immersion effects are 
explicitly accounted for is quite significant for the alcohols (the standard deviation drops from 1.44 to $0.97 \mathrm{mN} / \mathrm{m}$, Table 1). The comparison between the data and the predictions of the corrected SD model is given in Figure 5a, and between the two variants of the SD model - in S2. On the contrary, for acids, the results of the two variants of SD are practically equivalent in terms of standard deviation; the comparison with the data is given in S2, but the compensation between the osmotic and the immersion effect for acids is nearly complete and the theoretical lines are very close to those obtained previously in Ref. [23]. Figure 5b illustrates the results for $\mathrm{C}_{n} \mathrm{H}_{2 n+1} \mathrm{EO}_{6}$. As another test of our model, we compared available neutron reflection data for the adsorption of butanol, hexanol [66], and $\mathrm{C}_{12} \mathrm{H}_{25} \mathrm{EO}_{6}$ [67] with the adsorption isotherm (4)-(5) of the SD model, with $K_{\mathrm{a} 0}$ and $\beta_{\mathrm{osm}}$ as obtained from the fit of the tensiometric data. The comparison with the data for butanol is excellent, as shown in Figure 6. The adsorption data for hexanol and $\mathrm{C}_{12} \mathrm{H}_{25} \mathrm{EO}_{6}$ both show similar negative deviations from the theoretical line, discussed in $\mathrm{S} 2$.

Table 1. Adsorption parameters of 3 homologous series of surfactants on water, obtained by minimization of Eq (24) using the sticky disc model with $\beta$ given by Eq (20).

\begin{tabular}{|c|c|c|c|c|}
\hline homologous series & $\ln \left(K_{\mathrm{a} 0} / \mathrm{m}\right)^{\mathrm{c}}$ & $\alpha_{\mathrm{s}}\left[\AA^{2}\right]$ & $\begin{array}{c}\beta= \\
\beta_{\mathrm{vdW}}+\beta_{\mathrm{osm}} \mathrm{e}\end{array}$ & $\operatorname{dev}[\mathrm{mN} / \mathrm{m}]$ \\
\hline \multirow{2}{*}{$\mathrm{C}_{n} \mathrm{H}_{2 n+1} \mathrm{OH}^{\mathrm{a}}$} & -20.4 & \multirow{2}{*}{$\underline{16.5}^{\mathrm{d}}$} & $\begin{array}{c}\beta_{\mathrm{osm}}=0 \\
\beta_{\mathrm{vdW}, n}\end{array}$ & 1.44 \\
\hline & -20.2 & & $\begin{array}{c}\beta_{\mathrm{osm}}=0.98, \\
\beta_{\mathrm{vdW}, n-1}\end{array}$ & 0.97 \\
\hline \multirow{2}{*}{$\mathrm{C}_{n-1} \mathrm{H}_{2 n-1} \mathrm{COOH}^{\mathrm{a}}$} & -20.2 & \multirow{2}{*}{$\underline{18}^{\mathrm{d}}$} & $\begin{array}{c}\beta_{\mathrm{osm}}=0 \\
\beta_{\mathrm{vdW}, n}\end{array}$ & 0.93 \\
\hline & -20.1 & & $\begin{array}{c}\beta_{\mathrm{osm}}=0.84 \\
\beta_{\mathrm{vdW}, n-1}\end{array}$ & 0.90 \\
\hline \multirow{2}{*}{$\mathrm{C}_{n} \mathrm{H}_{2 n+1} \mathrm{EO}_{6}{ }^{\mathrm{b}}$} & -16.82 & 33.3 & $\begin{array}{c}\beta_{\mathrm{osm}}=0 \\
\beta_{\mathrm{vdW}, n}\end{array}$ & 0.67 \\
\hline & -16.89 & 34.3 & $\begin{array}{c}\beta_{\mathrm{osm}}=0.50 \\
\beta_{\mathrm{vdW}, n-1}\end{array}$ & 0.655 \\
\hline
\end{tabular}

a The data are for $n=3 \div 10$, average $T=21{ }^{\circ} \mathrm{C}$. ${ }^{\mathrm{b}}$ Data for $n=12$ and $14, T=25{ }^{\circ} \mathrm{C}$. ${ }^{\mathrm{c}}$ The transfer energy in the expression (21) for the adsorption constant $K_{\mathrm{a}}$ is fixed to $\Delta \mu_{\mathrm{CH}_{2}}=1.04 \times k_{\mathrm{B}} T$ [37]. ${ }^{\mathrm{d}} \mathrm{Value}$ of the hard-disc area calculated from crystallographic and collapse data of alcohols and acids, see the text. ${ }^{e}$ Fixed to the value predicted by Eq. (23), with $n$ or $n-1 \mathrm{CH}_{2}$ groups contributing to $\beta_{\mathrm{vdw}}$, with or without $\beta_{\text {osm. }}$ 


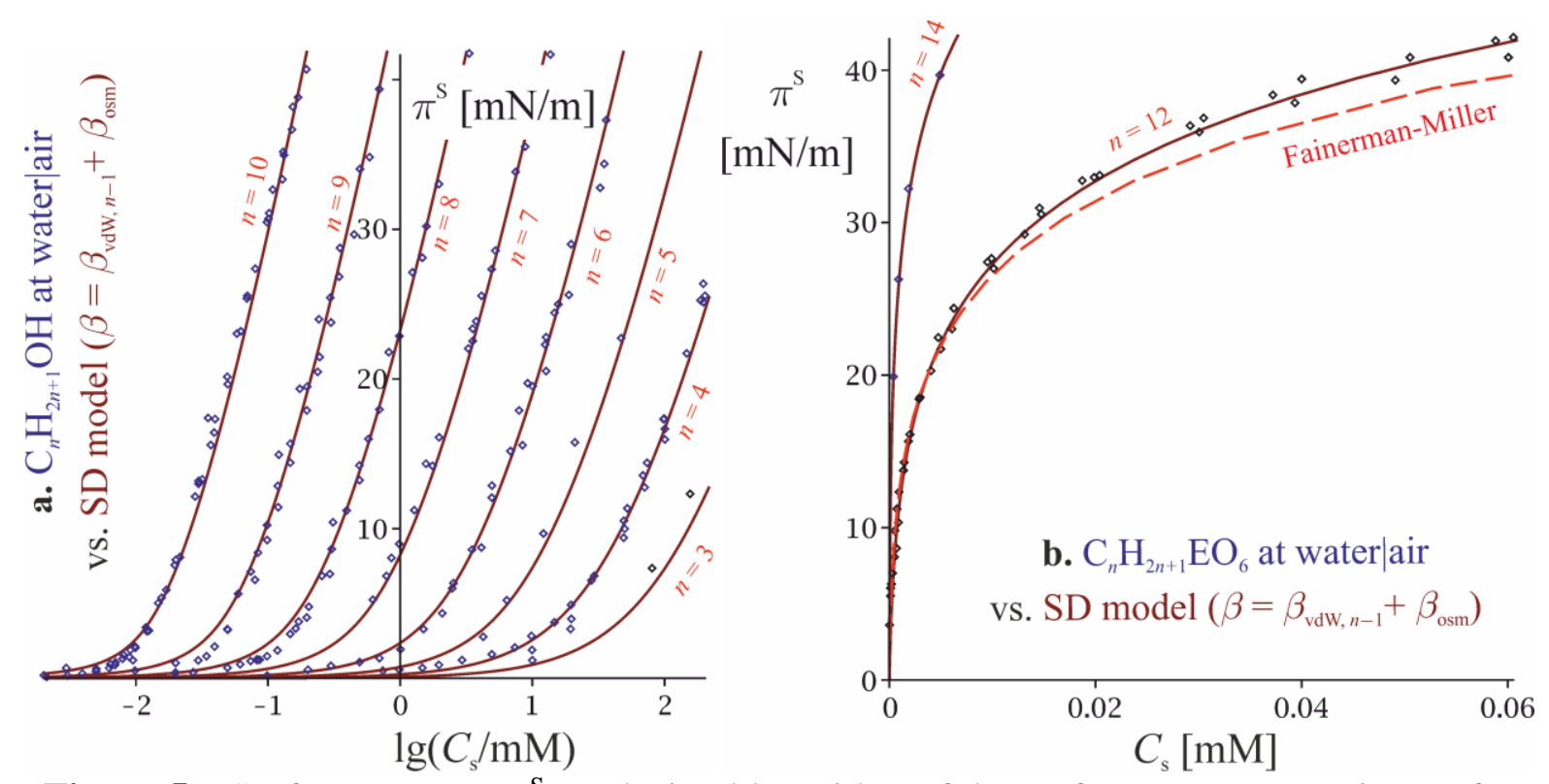

Figure 5a. Surface pressure $\pi^{\mathrm{S}}$ vs. decimal logarithm of the surfactant concentration $C_{\mathrm{s}}$ for alcohols at W|A. Lines stand for the SD model with attraction parameter $\beta=\beta_{\mathrm{osm}}+\beta_{\mathrm{vdW}, n-1}$ (corrected for the solvent effect and for one immersed methylene group, Eq (23)). Two adjustable parameters for the whole homologous series were determined: $\ln \left(K_{\mathrm{a} 0} / \mathrm{m}\right)=-20.2$ and $\beta_{\text {osm }}=0.98$. b. Surface pressure $\pi^{\mathrm{S}}$ vs. surfactant concentration $C_{\mathrm{s}}$ for $\mathrm{C}_{n} \mathrm{H}_{2 n+1} \mathrm{EO}_{6}$. Lines are the SD model with $\ln \left(K_{\mathrm{a} 0} / \mathrm{m}\right)=-16.9, \alpha_{\mathrm{s}}=34.3 \AA^{2}$, and $\beta_{\mathrm{osm}}=0.5$. The reorientation model of Fainerman and Miller [9] is plotted for comparison.

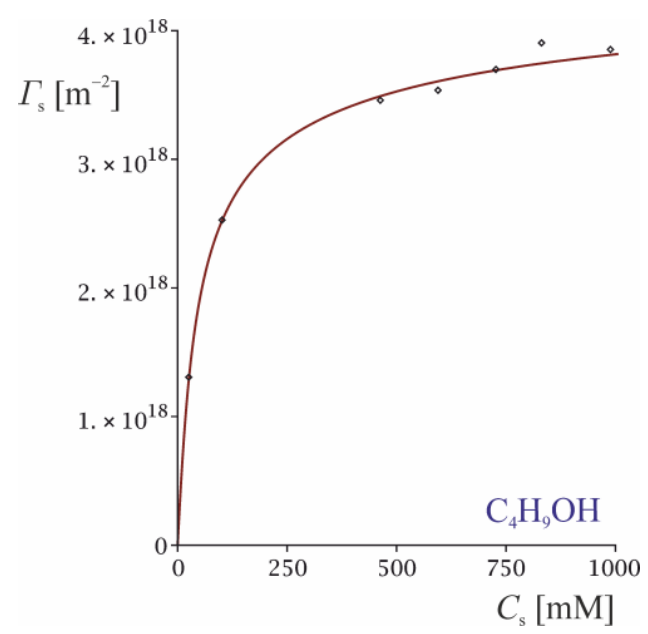

Figure 6. Adsorption of butanol at W|A vs. surfactant concentration $C_{\mathrm{s}}$. Points: neutron reflection data from Ref. [66]. Line: the SD adsorption isotherm (4)-(5), with the hard-disc area $\alpha_{\mathrm{s}}=16.5 \AA^{2}$, attraction parameter $\beta$ calculated from Eq (23) and adsorption constant $K_{\mathrm{a}}$ from Eq (21), with $K_{\mathrm{a} 0}$ and $\beta_{\text {osm }}$ from Table 1.

Let us now discuss the values we obtained for the adsorption parameters. The fit gives $\ln \left(K_{\mathrm{a} 0} / \mathrm{m}\right)=-20.2,-20.1$, and -16.9 for the alkanols, the acids and the hexaethoxylates, respectively (Table 1). On the other hand, the theoretical values computed through Ivanov's model $(22)$ are $\ln \left(K_{\mathrm{a} 0} / \mathrm{m}\right)=-20.3,-20.0$, and -16.9 . The agreement is remarkable, and shows that the dependence of the adsorption constant on the nature of the polar head group of the surfactant is indeed due to the term $\alpha_{\perp} \sigma_{0}$ in Eq (22). Therefore, the well-known tendency of $K_{\mathrm{a}}$ to increase with each ethyleneglycol group added (e.g., table 3.13 in Ref. [9]) seems to be related to the respective increase of the hard-disc area of the $\mathrm{EO}_{m}$ head group. 
The hard-disc area of the $\mathrm{C}_{n} \mathrm{H}_{2 n+1} \mathrm{EO}_{6}$ found from the fit is $34.3 \AA^{2}$ (Table 1). This is practically equivalent to the value $\alpha_{\mathrm{s}}=34.2 \AA^{2}$ following from the collapse area of Lange an Jeschke [46] discussed above.

Finally, let us discuss the two effects - from the solvent in the monolayer and from the immersion - on the values of the lateral attraction parameter. The values of $\beta$ that follow from Eq (23) for all studied surfactants are summarized in Table 2 in S2, where they are compared to the approximation $\beta=\beta_{\mathrm{vdW}, n}$ neglecting the two effects. Despite the compensation of effects, the difference between $\beta_{\mathrm{osm}}+\beta_{\mathrm{vdW}, n-1}$ and $\beta_{\mathrm{vdW}, n}$ is not small. With regard to the osmotic parameter, the fitted value in Table 1 found for alcohols $\left(\beta_{\mathrm{osm}}=0.98\right)$ corresponds, according to $\mathrm{Eq}(16)$, to $\psi_{\mathrm{w} 0}=0.77$; the value for the acids $\left(\beta_{\mathrm{osm}}=0.84\right)$ corresponds to $\psi_{\mathrm{w} 0}=0.61$, and $\beta_{\mathrm{osm}}=0.50$ for the ethyleneglycol ethers corresponds to $\psi_{\mathrm{w} 0}=0.36$. Clearly, $\beta_{\text {osm }}$ decreases as $\alpha_{\mathrm{s}}$ increases, in qualitative agreement with $\mathrm{Eq}(16)$, as illustrated in Figure 4. While the qualitative trend with $\alpha_{\mathrm{s}}$ and the order of magnitude of $\beta_{\mathrm{osm}}$ are very reasonable, quantitatively, there is a discrepancy. This is not surprising, in view of the limitations of the model, (i-iv) in Sec. 2.3. In addition, the uncertainty of the $\beta_{\text {osm }}$ values we obtained is not small. Our adsorption model is much more sensitive to $\alpha_{\mathrm{s}}$ and $\Delta \mu_{\mathrm{CH}_{2}}$ than to $\beta_{\mathrm{osm}}-$ a small change in the values of $\alpha_{\mathrm{s}}$ and $\Delta \mu_{\mathrm{CH}_{2}}$ has an effect on the adsorption behaviour comparable with the osmotic effect we consider. Yet, we use for them independent estimates that are not too accurate $\left(\Delta \mu_{\mathrm{CH}_{2}} / k_{\mathrm{B}} T=\right.$ $1.04 \pm 0.06 ; \alpha_{\mathrm{s}}=16.5 \pm 0.4 \AA^{2}$ for alcohols and $18.0 \pm 0.8$ for acids). If we vary these parameters within their uncertainty limits, the results for $\beta_{\text {osm }}$ will change significantly.

Extensive comparison of the SD theory with other models has been given previously [23]; one model we did not consider there (as only surfactants of relatively simple head groups were analysed) was the reorientation model of Fainerman and Miller [9]. This is a typical localized EoS based on Langmuir's. According to Fainerman and Miller, surfactants such as alkanol polyethoxylates have two stable conformations at the surface, corresponding to two populations of adsorbed molecules, characterized by two respective area parameters and two adsorption energies. Using the parameters for from table 3.13 of Ref. [9] in eqs. 3.3-3.9 there, we calculated the surface pressure and the adsorption of this surfactant as functions of the concentration. The result for the surface pressure is the dashed line in Figure 5b, and it stands below the experimental data. This is entirely due to the fact that Fainerman and Miller used different sources (including some at a different temperature), which do not agree well with each other. It is actually not a problem to fit with a 4-parametric model the tensiometric data in Figure 5b to within its experimental uncertainty. Moreover, in S2, it is shown that the comparison of the reorientation model with adsorption data directly obtained from neutron scattering is better than the one of the SD model. This only shows that when two models describe a dataset well, a small difference in the respective deviations is not a useful criterion to distinguish between them. What matters is how sensible are the parameter values. The area of the contracted state of the monolayer obtained in Ref. [9] is $49.3 \AA^{2}$; this is far bigger than the collapse area $\alpha_{\perp}=37.8 \AA^{2}$ obtained in Ref. [46], i.e. a situation that is difficult to explain occurs, where the monolayer can be experimentally compressed to values smaller than the theoretically possible limit. This paradox occurs every time when localized models (Langmuir, Frumkin's etc.) are applied to data for liquid interfaces, but disappears when the appropriate delocalized models are utilized [23]. In addition, the model of Fainerman and Miller ignores completely the lateral attraction between the hydrocarbon chains of $\mathrm{C}_{12} \mathrm{H}_{25} \mathrm{EO}_{6}$, while the direct computation via Eqs (7)-(8) would show a non-negligible value $\left(\beta_{\mathrm{vdW}, n-1}=0.93\right)$.

A unique feature of our model is that the knowledge of the value of $\beta_{\text {osm }}$ allows us to estimate the decrease of the amount of solvent in the surface layer through the isotherm (13) of the solvent. For example, for $\mathrm{C}_{n} \mathrm{H}_{2 n+1} \mathrm{EO}_{6}$, from the fitted values $\beta_{\text {osm }}=0.50$ and $\alpha_{\mathrm{s}}=34.3 \AA^{2}$, we find $\psi_{\mathrm{w} 0}=0.36$ through Eq (16). Using this $\psi_{\mathrm{w} 0}$ in the adsorption isotherm of the solvent 
(13) for the hard-disc mixture model, together with the SD adsorption isotherm (4)-(5) for the surfactant, we can compute the surface fractions of water and surfactant as functions of the surfactant concentration. This is done in Figure 7. The results for the alcohols are similarly dealt with in S2. Due to the limitations of the model (Sec. 2.3), the result for the water content $\psi_{\mathrm{w}}$ and the total coverage $\psi_{\mathrm{w}}+\psi_{\mathrm{s}}$ is probably a crude approximation $\left(\psi_{\mathrm{s}}\right.$, on the other hand, must be accurate since any reasonable interpolation of the tensiometric data will produce the correct surfactant adsorption). Nevertheless, the trends observed are reasonable:

(i) the solvent surface fraction drops and approaches zero as more surfactant adsorbs;

(ii) the total coverage (total density of the monolayer) increases with the increase of $\psi_{\mathrm{s}}$. Note that the hard-disc mixture model predicts that the monolayer is of total coverage significantly lower than 1 , and has a significant compressibility. In contrast, both approaches that Frumkin cited in defence of the Langmuir model $[1,6]$ assume that the monolayer is incompressible and $\psi_{\mathrm{w}}+\psi_{\mathrm{s}}=1$; the same is valid for the EoS (17) of Chattoraj and Birdi [18]. The expulsion of water from the monolayer we predict has been demonstrated with direct neutron scattering measurements (e.g., fig. 9 in Ref. [68]); quantitatively, however, the experiment $[68,67]$ seems to show water content in the dense monolayer (near the critical micelle concentration) that is higher than the prediction of the hard-disc mixture model. The probable reasons are the association between water and $\mathrm{C}_{n} \mathrm{H}_{2 n+1} \mathrm{EO}_{6}$ [67], neglected by the SD model, and the failure of the monolayer approximation (several layers of water can fit in a layer of $\mathrm{EO}_{6}$ polar head groups).

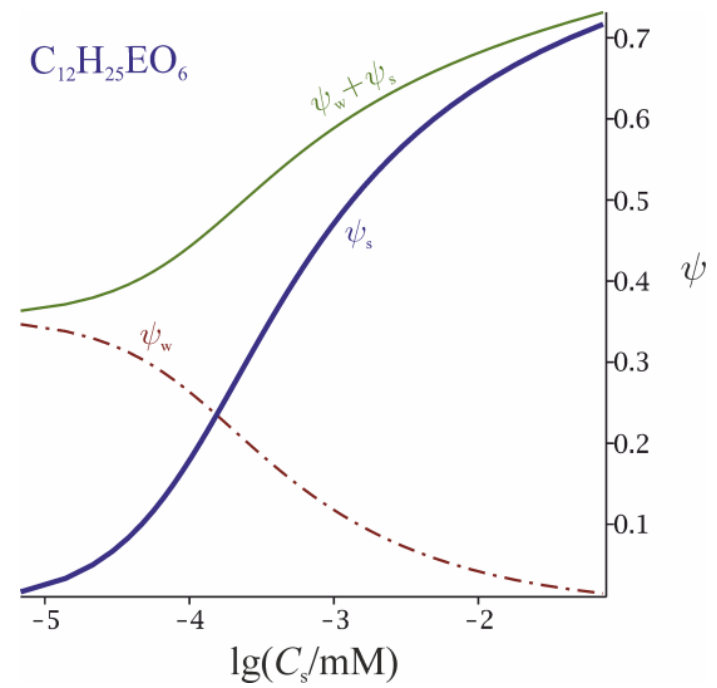

Figure 7. Surface coverage with solvent $\left(\psi_{\mathrm{w}}=\alpha_{\mathrm{w}} \Gamma_{\mathrm{w}}\right)$, surfactant $\left(\psi_{\mathrm{s}}=\alpha_{\mathrm{s}} \Gamma_{\mathrm{s}}\right)$, and total $\left(\psi_{\mathrm{w}}+\psi_{\mathrm{s}}\right)$ vs. the logarithm of surfactant concentration in the bulk phase for hexaethylenglycol monododecyl ether. Eq (13) is used to compute $\psi_{\mathrm{w}}$ and Eqs (4)\&(5) are used to compute $C_{\mathrm{s}}$ for a set of values of $\psi_{\mathrm{s}}$ to plot these curves.

\section{Discussion and conclusions}

Our work develops, to our knowledge, the first comprehensive delocalized model of adsorption of surfactant at a liquid surface with explicit account for the presence of solvent molecules in the surfactant layer. To do this, we utilized the equation of state of Lebowitz et al. for hard-disc mixtures [27] and the approximation for a surface monolayer [26]. 
We have shown that the osmotic effect due to the solvent molecules corresponds to an effective cohesion in the monolayer, characterized by an osmotic lateral attraction parameter $\beta_{\mathrm{osm}}$. This $\beta_{\mathrm{osm}}$ has been explicitly related to the density $\psi_{\mathrm{w} 0}$ of the surface layer of the neat solvent surface and the hard-disc area ratio $r=\alpha_{\mathrm{s}} / \alpha_{\mathrm{w}}$, Eq (16). We have demonstrated that the 1-component sticky disc model with $\beta$ set to $\beta_{\text {osm }}$ approximates well the more advanced 2component LHP model, Figure 3.

We further proposed a formula for the lateral attraction parameter, Eq (23), that involves the van der Waals attraction between the hydrocarbon chains (the main contribution to $\beta$ ), the osmotic cohesion effect, and the contribution due to the immersion of the methylene group adjacent to the polar head group. We demonstrated that this formula agrees very well with the experimental data for three homologous series of surfactants at W|A, Table 1 and Figure 5.

The results from our work explain several experimental observations that are puzzling otherwise:

(i) the 1-component HFL and SD models were previously found successful in describing the adsorption behaviour of numerous surfactants at W|A interface [23], despite the fact that they neglect the solvent molecules. The explanation is the nearly complete cancelation of the osmotic cohesion effect from the solvent and the effect from the immersion in the aqueous phase of the $\mathrm{CH}_{2}$ group adjacent to the polar head-group, cf. Section 3.

(ii) An interesting prediction from the model is that, if the chemical potential of water is decreased via addition of surface-inactive electrolyte, this will result in smaller $\psi_{\mathrm{w} 0}$ and, as a consequence, in smaller osmotic attraction. Indeed, the monolayer cohesion has been observed to decrease at concentration of $\mathrm{NaF}$ or $\mathrm{NaCl}$ of the order of $1 \mathrm{M}$ and more (e.g., Fig. 5 in Ref. [41]). However, this effect is complicated by the direct screening of the van der Waals attraction between the hydrocarbon tails by the salt.

(iii) The small $n$-independent lateral attraction parameter found previously for acids and alcohols adsorbed at water|oil interfaces (table 2 in Ref. [23]) is most probably also of osmotic origin.

With regard to the last point, it is not clear how two solvents in two surface layers (one in the aqueous and another in the oil phase) would contribute to the adsorption at water|oil interfaces. Nevertheless, our results predict an interesting dependence of the lateral attraction parameter on the size of the oil molecule: according to Eq (16), large solvent molecules must lead to large effective attraction (large $\beta_{\text {osm }}$ ). We hope we will be able to test this prediction in future.

Acknowledgements. RS would like to acknowledge the funding and technical support from BP through the BP International Centre for Advanced Materials (BP-ICAM) which made this research possible. Discussions with Prof. Stuart Clarke have helped us to improve this work.

\section{References}

The publication contains supplementary materials: S1. Symbols, abbreviations, and definitions. S2. Additional data. S3. Limit of the adsorption isotherms at $\psi_{\mathrm{w}} \rightarrow 0$ (dry monolayer). S4. The case of concentrated bulk solution. S5. Derivation of the activity coefficients of the LHP model. S6. Excel spreadsheet with the experimental data.

1. A.N. Frumkin (editor), The Basic Problems in Modern Theoretical Electrochemistry: Works from the $14^{\text {th }}$ Meeting of the International Committee of Electrochemical Thermodynamics and Kinetics. Mir; 1965, in Russian. 
2. F.P. Buff, F.H. Stillinger. Statistical mechanical theory of double-layer structure and properties. J. Phys. Chem. 39 (1963) 1911.

3. R. Parsons, J. Electroanal. Chem. 7 (1964) 136-152.

4. F.P. Buff, F.H. Stillinger. In A.N. Frumkin (editor), Basic Problems of Modern Theoretical Electrochemistry. Mir; 1965, in Russian.

5. E. Helfand, H.L. Frisch, J.L. Lebowitz, J. Chem. Phys. 34 (1961) 1037.

6. A.N. Frumkin, J. Electroanal. Chem. 7 (1964) 152-155.

7. R. Parsons, Russ. J. Electrochem. 37 (2001) 647-652.

8. R. Parsons, J. Electroanal. Chem. 8 (1964) 93-98.

9. V.B. Fainerman, R. Miller, E.V. Aksenenko, A.V. Makievski. In V.B. Fainerman, D. Möbius, R. Miller (editors), Surfactants - Chemistry, Interfacial Properties, Applications. Elsevier; 2001. Chapter 3.

10. K. Lunkenheimer, W. Barzyk, R. Hirte, R. Rudert, Langmuir 19 (2003) 6140.

11. J. Lucassen, D. Giles. J. Chem. Soc., Faraday Trans. 171 (1975) 217-232.

12. S.-Y. Lin, Y.-C. Lee, M.-W. Yang, H.-S. Liu. Langmuir 19 (2003) 3164-3171.

13. Lj.M. Vračar, D.M. Dražić, Corrosion Science 44 (2002) 1669-1680.

14. V.S. Markin, M.I. Volkova-Gugeshashvili, A.G. Volkov. J. Phys. Chem. B 110 (2006) 11415-11420.

15. C.E. Morgan, C.J.W. Breward, I.M. Griffiths, P.D. Howell, J. Penfold, R.K. Thomas, I. Tucker, J.T. Petkov, J.R.P. Webster. Langmuir 28 (2012) 17339-17348.

16. J. Lawrence, R. Parsons, J. Phys. Chem. 73 (1969) 3577.

17. T. Smith, J. Colloid Interface Sci. 23 (1967) 27.

18. D.K. Chattoraj, K.S. Birdi, Adsorption and the Gibbs Surface Excess. Plenum press; 1984. Chapters 5\&6.

19. I.B. Ivanov, K.P. Ananthapadmanabhan, A. Lips. Adv. Colloid Interface Sci. 123-126 (2006) 189.

20. T.D. Gurkov, I.B. Ivanov. In Proc. 4th World Congress on Emulsions, Lyon, 2006, p. 509.

21. I.B. Ivanov, K.D. Danov, D. Dimitrova, M. Boyanov, K.P. Ananthapadmanabhan, A. Lips, Colloids Surfaces A 354 (2010) 118

22. R.I. Slavchov, S.I. Karakashev, I.B. Ivanov. In L. Römsted (editor), Surfactant Science and Technology: Retrospects and Prospects. Taylor and Francis, LLC; 2014. Chapter 2.

23. R.I. Slavchov, I.B. Ivanov, Soft Matter 13 (2017) 8829-8848.

24. T. Söderlund, J.-M.I. Alakoskela, A.L. Pakkanen, P.K.J. Kinnunen, Biophys. J. 85 (2003) 2333 -2341.

25. J.N. Israelachvili, Intermolecular and Surface Forces, $3^{\text {rd }}$ ed. Academic Press; 2011.

26. R. Defay, I. Prigogine. Surface tension and adsorption. Longmans; 1966.

27. J.L. Lebowitz, E. Helfand, E. Praestgaard. Scaled particle theory of fluid mixtures. J. Chem. Phys. 43 (1965) 774

28. M. Mezger, F. Sedlmeier, D. Horinek, H. Reichert, D. Pontoni, H. Dosch, J. Am. Chem. Soc. 132 (2010) 6735.

29. Analogous to the virial expansion of the 3D osmotic pressure: T.L. Hill, An Introduction to Statistical Thermodynamics. Addison-Wesley; 1962 (sec. 19-1).

30. E.H. Lucassen-Reynders, M. van den Tempel, Proc. Ivth Int. Cong. Surface Active Substances, Brussels, 1964, vol. 2, p. 779.

31. M.A. Volmer, P. Mahnert, Z. Phys. Chem., 1925, 115, 236.

32. J.H. De Boer, The Dynamical Character of Adsorption. Clarendon Press; 1953.

33. C. Tanford, The Hydrophobic Effect. Wiley; 1980.

34. P. Kruglyakov, Hydrophile-Lipophile Balance of Surfactants and Solid Particles. Elsevier; 2000.

35. R.A. Pierotti, Chem. Rev. 76 (1976) 717-726

36. R.I. Slavchov, I.M. Dimitrova, T. Ivanov, J. Chem. Phys. 143 (2015) 154707

37. R.I. Slavchov, I.M. Dimitrova, I.B. Ivanov. In R.G. Rubio, Y.S. Ryazantsev, V.M. Starov, G.X. Huang, A.P. Chetverikov, P. Arena, A.A. Nepomnyashchy, A. Ferrús, E.G. Morozov (editors), Without Bounds: a Scientific Canvas of Nonlinearity and Complex Dynamics. Springer-Verlag; 2013, p. 199

38. W. Hückel, Theoretical Principles of Organic Chemistry, Vol. II, p. 435. Elsevier; 1958.

39. A.I. Kitaigorodskii, Organic Chemical Crystallography. Consultant Bureau; 1961.

40. A. Gericke, J. Simon-Kutscher, H. Hühnerfuss. Langmuir 9 (1993) 3115-3121

41. R.V. Peshkova, I.L. Minkov, R. Tsekov, R.I. Slavchov, Langmuir 32 (2016) 8858-8871.

42. I. Langmuir, J. Chem. Phys., 1933, 1, 756.

43. G. Jura, W.D. Harkins, J. Chem. Phys., 1944, 12, 113.

44. N.K. Adam, The Physics and Chemistry of Surfaces. Clarendon Press; 1941.

45. A.D. Bond. New. J. Chem. 28 (2004) 104-114

46. H. Lange, P. Jeschke, in M.J. Schick (Ed.), Nonionic surfactants. Physical chemistry, Marcel Dekker Inc. New York, 1987. 
47. J.T. Davies, E. Rideal, Interfacial Phenomena. Academic Press; 1963. Chapters 4\&5.

48. K.D. Danov, P.A. Kralchevsky, K.P. Ananthapadmanabhan, A. Lips, J. Colloid Interface Sci., 2006, 300, 809

49. A.J. Kumpulainen, C.M. Persson, J.C. Eriksson, E.C. Tyrode, C.M. Johnson, Langmuir, 2005, 21,305

50. J.R. Hommelen, J. Colloid Science 14 (1959) 385.

51. S.Y. Lin, K. McKeigue, C. Maldarelli, Langmuir 7 (1991) 1055.

52. S.Y. Lin, T.L. Lu, W.B. Hwang, Langmuir 10 (1994) 3442.

53. S.Y. Lin, Y.C. Lee, M.W. Yang, H.S. Liu, Langmuir 19 (2003) 3164.

54. R. Vochten, G. Petre, J. Colloid lnterface Sci. 42 (1973) 320.

55. V.B. Fainerman, S.V. Lylyk, Koll. Zh. 45 (1983) 500.

56. C.C. Addison, J. Chem. Soc. (1945) 98.

57. M. Aratono, S. Uryu, Y. Hayami, K. Motomura, R. Matuura, J. Colloid Interface Sci. 98 (1984) 33.

58. W. Barzyk, K. Lunkenheimer, P. Warszyński, B. Jachimska, A. Pomianowski. Colloids Surf. A 443 (2014) $515-524$

59. R. Wüstneck, R. Miller, Colloids Surf. 47 (1990) 15.

60. K. Lunkenheimer, R. Hirte, J. Phys. Chem. 96 (1992) 8683.

61. M. Aratono, S. Uryu, Y. Hayami, K. Motomura, R. Matuura, J. Colloid Interface Sci. 98 (1984) 33.

62. V.D. Dolzhikova, Y.V. Goryunov, B.D. Summ, Colloid J. 44 (1982) 560.

63. K. Malysa, R. Miller, K. Lunkenheimer, Colloids Surf. 53 (1991) 47.

64. F. Suarez, C.M. Romero, J. Chem. Eng. Data 2011, 56, 1778-1786

65. N. Nishikido, T. Takahara, H. Kobayashi, M. Tanaka, Bull. Chem. Soc. Jpn. 55 (1982) 3085-3088.

66. Z.X. Li, J.R. Lu, R.K. Thomas, A.R. Rennie, J. Penfold. J. Chem. Soc., Faraday Trans. 92 (1996) 565572 .

67. J.R. Lu, Z.X. Li, R.K. Thomas, E.J. Stalpes, I. Tucker, J. Penfold. J. Phys. Chem. 97 (1993) 8012-8020.

68. J.R. Lu, T.J. Su, Z.X. Li, R.K. Thomas, E.J. Stalpes, I. Tucker, J. Penfold. J. Phys. Chem. B 101 (1997) 10332-10339. 


\title{
Effective osmotic cohesion due to solvent molecules in a delocalized adsorbed monolayer
}

Supplementary information

\author{
Radomir I. Slavchov ${ }^{1}$, Ivan B. Ivanov ${ }^{2}$ \\ ${ }^{1}$ Department of Chemical Engineering \\ ${ }^{2}$ Laboratory of Chemical Physics \\ University of Cambridge \\ and Engineering \\ West Site, Philippa Fawcett Drive \\ Faculty of Chemistry and Pharmacy \\ Cambridge, CB3 0AS \\ Sofia University \\ United Kingdom \\ 1 J. Bourchier, 1164 Sofia, Bulgaria \\ E-mail: ris26@cam.ac.uk \\ E-mail: ii@lcpe.uni-sofia.bg
}

\section{S1. Symbols, abbreviations, and definitions}

$B_{2} \quad$ second virial coefficient of the surfactant monolayer

$C_{\mathrm{s}} \quad$ concentration of surfactant in the bulk phase

$K_{\mathrm{a}} \quad$ adsorption constant

$k_{\mathrm{B}} \quad$ Boltzmann constant

$L_{\mathrm{CH}_{2}} \quad$ London constant for the $\mathrm{CH}_{2}-\mathrm{CH}_{2}$ interaction

$l_{\mathrm{CH}_{2}} \quad$ length of a $\mathrm{CH}_{2}$ group along the hydrophobic chain

$n$ number of methylene groups in the hydrocarbon chain of the surfactant

$R_{\beta} \quad$ the attraction term in the SD model, Eq (3)

$R_{\mathrm{S}} \quad$ hard-disc radius of the surfactant

$r \quad \equiv \alpha_{\mathrm{s}} / \alpha_{\mathrm{w}}$, surfactant/solvent hard-disc area ratio

$T$ temperature

$u_{\text {attr }} \quad$ binary attraction potential between adsorbed surfactant molecules

$V_{\mathrm{w}} \quad$ molecular volume of the solvent (molar volume divided by Avogadro's number)

$v_{\mathrm{w}} \quad$ hard sphere volume

$A_{\mathrm{w}} \quad$ molecular area at the neat surface, $1 / \Gamma_{\mathrm{w} 0}$

$\alpha \perp \quad$ collapse/crystallographic are of the surfactant molecule

$\alpha_{\mathrm{s}} \quad$ hard-disc area of the surfactant molecule

$\alpha_{\mathrm{w}} \quad$ hard-disc area of the solvent molecule

$\beta \quad$ lateral attraction parameter, Eqs (7) or (20)

$\beta_{\text {osm }} \quad$ effective contribution of the osmotic effect to $\beta, \mathrm{Eq}$ (16)

$\Gamma_{\mathrm{s}} \quad$ adsorption of surfactant

$\Gamma_{\mathrm{w}} \quad$ surface concentration of solvent in the surface layer

$\Gamma_{\mathrm{w} 0} \quad$ surface concentration of solvent in the surface layer for the neat surface (at $\psi_{\mathrm{s}}=0$ )

$\gamma_{\mathrm{s}} \quad$ surface activity coefficient of the surfactant

$\gamma_{\mathrm{s} 0} \quad$ surface activity coefficient of the surfactant at the neat surface

$\gamma_{\mathrm{w}} \quad$ surface activity coefficient of the solvent 
$\gamma_{\mathrm{w} 0} \quad$ surface activity coefficient of the solvent in the absence of surfactant

$\Delta \mu_{\mathrm{CH}_{2}}$ free energy of transfer of a $\mathrm{CH}_{2}$ from air to water, $\Delta \mu_{\mathrm{CH}_{2}} / k_{\mathrm{B}} T=1.04$.

$\pi^{\mathrm{S}} \quad$ surface pressure of the monolayer, $\pi^{\mathrm{S}} \equiv \sigma_{0}-\sigma$

$\rho \quad$ integration variable in Eq (7) (distance between surfactant molecules)

$\sigma \quad$ surface tension of the monolayer

$\sigma_{0} \quad$ surface tension of the neat solvent surface (at $\psi_{\mathrm{s}}=0$ )

$\psi_{\mathrm{s}} \equiv \alpha_{\mathrm{s}} \Gamma_{\mathrm{s}}$, the area fraction covered by surfactant

$\psi_{\mathrm{w}} \equiv \alpha_{\mathrm{w}} \Gamma_{\mathrm{w}}$, the area fraction of solvent in the surface layer

$\psi_{\mathrm{w} 0} \equiv \alpha_{\mathrm{w}} \Gamma_{\mathrm{w} 0}$, the area fraction of solvent in the surface layer of the neat solvent (at $\left.\psi_{\mathrm{s}}=0\right)$

1D one-dimensional

2D two-dimensional

3D three-dimensional

$\mathrm{CH}_{2}$ methylene group

$\mathrm{CH}_{3}$ methylene group

EoS equation of state

HFL the model of a 1-component hard-disc fluid by Helfand, Frisch and Lebowitz

LHP the model of a 2-component hard-disc fluid of Lebowitz, Helfand and Praestgaard

SD the sticky disc adsorption model

W|A water|air interface

Definitions. A localized equation of state (based on a lattice, or Flory-Huggins models) are those that assume distribution of molecules at pre-existing sites or lattice nodes. Among the popular adsorption models, such are the models of Langmuir, Frumkin, Schuchowitsky, Fainerman-Miller etc. The adsorbed molecules are assumed to be localized in a 3D potential well disallowing them to move freely in direction lateral to the surface (a jump from one site to another has an energetic barrier that exceeds $k_{\mathrm{B}} T$ ). These models are suitable for adsorption at solid surfaces having adsorption sites.

The delocalized equations of state are based on a description of the surface monolayer as a non-ideal fluid, where the molecules are free to move in lateral direction (the barrier for lateral diffusion is below $k_{\mathrm{B}} T$ ). Examples for such models are those of Volmer, van der Waals, Chattoraj-Birdi (which are essentially models of a 1D liquid [23]), HFL, sticky disc (models of a 2D liquid).

That the solvent effect is osmotic follows from the definition of the surface pressure of a monolayer as the surface excess of the osmotic pressure - the surface pressure can be considered as the osmotic pressure in a monolayer of water diluted with surfactant times the monolayer's effective thickness (e.g., Ref. [18]). A single component EoS assumes instead the monolayer behaves as a non-ideal gas and the surface pressure is the pressure of this gas. Frumkin's criticism can be formulated as follows: the pressure of a one-component imperfect gas is a worse approximation for $\pi^{S}$ of a monolayer than the osmotic pressure in a mixture of solvent and adsorbent in a lattice.

The lateral attraction parameter $\beta$ of a 1 -component imperfect gas is defined via the $2^{\text {nd }}$ virial coefficient of this gas, Eq (6). The lateral attraction parameter of a 2-component mixture is defined similarly via the osmotic virial coefficient.

Cohesive monolayers, as a phenomenological concept, are monolayers that show negative deviations from the ideal gas equation of state, and have a characteristic inflection in their EoS. A monolayer is cohesive when $\beta>2$ [23].

An adsorption model consists of (i) an adsorption isotherm of the surfactant (the condition for chemical equilibrium of the surface active species at the surface and in the bulk relating $\Gamma_{\mathrm{s}}$ 
and $C_{\mathrm{s}}$ ) and (ii) an equation of state ( $\pi^{\mathrm{S}}$ vs. $\Gamma_{\mathrm{s}}$ ), which are related to each other via the Gibbs adsorption equation. For the explicit dependence of $\pi^{\mathrm{S}}$ on $C_{\mathrm{s}}$ (combining the adsorption isotherm and the EoS), we use the term surface pressure isotherm. A 2-component model would have also (iii) an adsorption isotherm for the solvent - e.g., Eq (13) of LHP. All models in the literature we are aware of (e.g., [6,18]) assume monolayer incompressibility $\left(\psi_{\mathrm{s}}+\psi_{\mathrm{w}}=1\right)$, in which case the isotherm of the solvent reads simply $\psi_{\mathrm{w}}\left(C_{\mathrm{s}}\right)=1-\psi_{\mathrm{s}}\left(C_{\mathrm{s}}\right)$. The incompressibility assumption is, however, not realistic - for example, in the absence of surfactant it leads to the impossible $\psi_{\mathrm{w} 0}=1$ for the neat surface (cf. the discussion following Eq (14)).

We call the quantity $1 / \Gamma_{\mathrm{s}}$ area per surfactant molecule.

The crystallographic area per molecule $\alpha_{\perp}$ is the cross-sectional area per surfactant molecule in a crystal structure for an axis orientation that minimizes this area (for simple surfactants, this is usually the one normal to the hydrocarbon chain). A straightforward way to obtain an accurate value of this quantity is to consider the molar volume $V_{\mathrm{s}}$ in a crystal lattice of a homologous series of surfactants, and to determine the increment $\mathrm{d} V_{\mathrm{s}} / \mathrm{d} n$ with the addition of one $\mathrm{CH}_{2}$ group in the hydrocarbon chain (as the slope of the linear dependence of $V_{\mathrm{s}}$ on $n$ ). The ratio between $\mathrm{d} V_{\mathrm{s}} / \mathrm{d} n$ and the length of a $\mathrm{CH}_{2}$ group along the hydrophobic chain $\left(l_{\mathrm{CH}_{2}}=\right.$ $1.26 \AA$ ) is giving $\alpha_{\perp}$.

The collapse area is an experimental quantity obtained for insoluble monolayers in a Langmuir trough compressed until the monolayer becomes instable and crystals, multilayer structure or lenses are formed. The collapse area is, as a rule, by $0-5 \%$ larger than the crystallographic area [42].

The hard-disc area of a surfactant, $\alpha_{\mathrm{S}}=\pi R_{\mathrm{s}}^{2}$, where $R_{\mathrm{S}}$ is the radius of the hard disc (i.e. $2 R_{\mathrm{S}}$ is the van der Waals radius of the surfactant molecules when aligned parallel to each other). The hard-disc area is related to the crystallographic area $\alpha_{\perp}$ through a geometric factor that is usually close to 1.1 (it is 1.10 for hexagonal packing). For molecules of cross-section that is elliptic, the area of the ellipse can be approximately used (by analogy with a similar result for the 3D hard-sphere and hard-ellipsoid fluids).

Position of the Gibbs surface and relation of the surface concentration of water to the adsorption (surface excess) of water. Let $z=0$ correspond to the location of the monolayer (the average location of the centre of mass of the molecules in it). The model illustrated in Figure 1 can be represented with the following distribution of the water molecules

$$
C_{\mathrm{w}}(z)=\sum \Gamma_{\mathrm{w}, i} \delta\left(z-z_{i}\right),
$$

where $\Gamma_{\mathrm{w}, i}$ is molecules per unit area in the $i^{\text {th }}$ layer, $z_{i}$ is the location of the $i^{\text {th }}$ layer, $\delta$ is the Dirac function, and $z$ is the normal coordinate. We assume that the distance between two layers is $d_{\mathrm{w}}=\left(V_{\mathrm{w}}\right)^{1 / 3}$, with the exception of the distance between the first two layers, which is increased to $d_{1}>d_{\mathrm{w}}$. Therefore, the locations of the layers are explicitly given by

$$
z_{1}=0 ; \quad z_{i}=d_{1}+d_{\mathrm{w}}(i-2) \text { for } i \geq 2 .
$$

The density per unit area in each layer is assumed to be

$$
\Gamma_{\mathrm{w}, 1}=\psi_{\mathrm{w}} / \alpha_{\mathrm{w}} ; \quad \Gamma_{\mathrm{w}, i}=1 / A_{\mathrm{w}}=V_{\mathrm{w}}^{-2 / 3} \text { for } i \geq 2 .
$$

The adsorption corresponding to the model (25)-(26) at the surface where the monolayer is located $(z=0)$ follows straight from the its Gibbs definition as an excess:

$$
\Gamma_{\mathrm{w}, z=0}=\int_{-\infty}^{0}\left[C_{\mathrm{w}}(z)-1 / V_{\mathrm{w}}\right] \mathrm{d} z+\int_{0}^{\infty} C_{\mathrm{w}}(z) \mathrm{d} z=\Gamma_{\mathrm{w}}-\left(d_{1}-d_{\mathrm{w}} / 2\right) / V_{\mathrm{w}} .
$$

For any other choice of the location of the surface of discontinuity, $z^{\mathrm{S}}$, the adsorption of water will be

$$
\Gamma_{\mathrm{w}, z=z^{\mathrm{s}}}=\int_{-\infty}^{z^{\mathrm{s}}}\left[C_{\mathrm{w}}(z)-1 / V_{\mathrm{w}}\right] \mathrm{d} z+\int_{z^{\mathrm{s}}}^{\infty} C_{\mathrm{w}}(z) \mathrm{d} z=\Gamma_{\mathrm{w}, z=0}-z^{\mathrm{s}} / V_{\mathrm{w}} .
$$


Similar relations hold for the adsorption of the surfactant:

$$
\begin{gathered}
\Gamma_{\mathrm{s}, z=0}=\Gamma_{\mathrm{s}}-\left(d_{1}-d_{\mathrm{w}} / 2\right) C_{\mathrm{s}}, \\
\Gamma_{\mathrm{s}, z=z^{\mathrm{s}}}=\Gamma_{\mathrm{s}, z=0}-z^{\mathrm{s}} C_{\mathrm{s}} .
\end{gathered}
$$

From Eqs (27)-(29), it follows that the Gibbs adsorptions are precisely equal to the surface densities $\Gamma_{\mathrm{w}}$ and $\Gamma_{\mathrm{s}}$ at one particular surface located at

$$
z^{\mathrm{s}}=-\left(d_{1}-d_{\mathrm{w}} / 2\right) \text {. }
$$

If the distance between the first two layers is $d_{1}=d_{\mathrm{w}}$, as in Figure 1, then our discontinuity surface of choice is at $z^{\mathrm{S}}=-d_{\mathrm{w}} / 2$, i.e. precisely between these two layers. These results are actually valid for any model that has a homogeneous solution below the surface (30), and a monolayer above it (i.e. for models in which the whole surface inhomogeneity is located in the surface monolayer, see also Ref. [26]).

The Gibbs equimolecular surface is the one that corresponds to $\Gamma_{\mathrm{w}, \mathrm{eqm}}=0$, and its location is, from Eqs (27)\&(28),

$$
z^{\mathrm{s}}=V_{\mathrm{w}} \Gamma_{\mathrm{w}, z=0}=V_{\mathrm{w}} \Gamma_{\mathrm{w}}-\left(d_{1}-d_{\mathrm{w}} / 2\right) .
$$

The respective Gibbs adsorption $\Gamma_{\mathrm{s} \text {,eqm }}$ of the surfactant (the one in $\Gamma_{\mathrm{s} \text {,eqm }}=\mathrm{d} \pi^{\mathrm{s}} / \mathrm{d} \mu_{\mathrm{s}}$ ) differs from $\Gamma_{\mathrm{s}}$ - from Eqs (29)\&(31), it follows that

$$
\Gamma_{\mathrm{s}, \text { eqm }}=\Gamma_{\mathrm{s}}-V_{\mathrm{w}} \Gamma_{\mathrm{w}} C_{\mathrm{s}} .
$$

According to this equation, the difference between the Gibbs adsorption $\Gamma_{\mathrm{s} \text {,eqm }}$ and the density $\Gamma_{\mathrm{s}}$ of the surfactant in the surface layer is only important at high concentrations and low surface activity. For example, for dilute solutions, $\Gamma_{\mathrm{s}}=K_{\mathrm{a}} C_{\mathrm{s}}$, and the correction for the shift of the Gibbs surface is unimportant if $K_{\mathrm{a}} \gg V_{\mathrm{w}} \psi_{\mathrm{w} 0} / \alpha_{\mathrm{w}}$; the value of $V_{\mathrm{w}} \psi_{\mathrm{w} 0} / \alpha_{\mathrm{w}}$ is of the order of 1.5$3 \AA$ (cf. the estimates following Eq (14)). The value of $K_{\mathrm{a}}$ for the shortest homologues we study (propanol and propanoic acid) is $400 \AA$. Therefore, for all surfactants we study, $\Gamma_{\mathrm{s}}$ can be safely assumed to be equal to the Gibbs adsorption of the surfactant.

\section{S2. Additional data}

Let us compare in more detail the two variants of the SD model we are using (the approximate SD model from Ref. [22] with $\beta=\beta_{\mathrm{vdW}, n}$, and the improvement proposed here with Eq (23) for $\beta_{\mathrm{vdW}, n-1}+\beta_{\mathrm{osm}}$, accounting for the solvent molecules in the monolayer and the $\mathrm{CH}_{2}$ group remaining immersed in the aqueous phase) for the case where the difference is greatest - the alcohols, which have the smallest $\alpha_{\mathrm{s}}$ of the surfactants we study. In Figure 8, surface tension data for the lowest and the highest alcohol homologues we studied are given, for which the difference between the models is most pronounced. The lines correspond to the two sets of parameters for alcohols in Table 1. It is seen that the improved model for $\beta$ leads to improved coincidence with the experiment. 


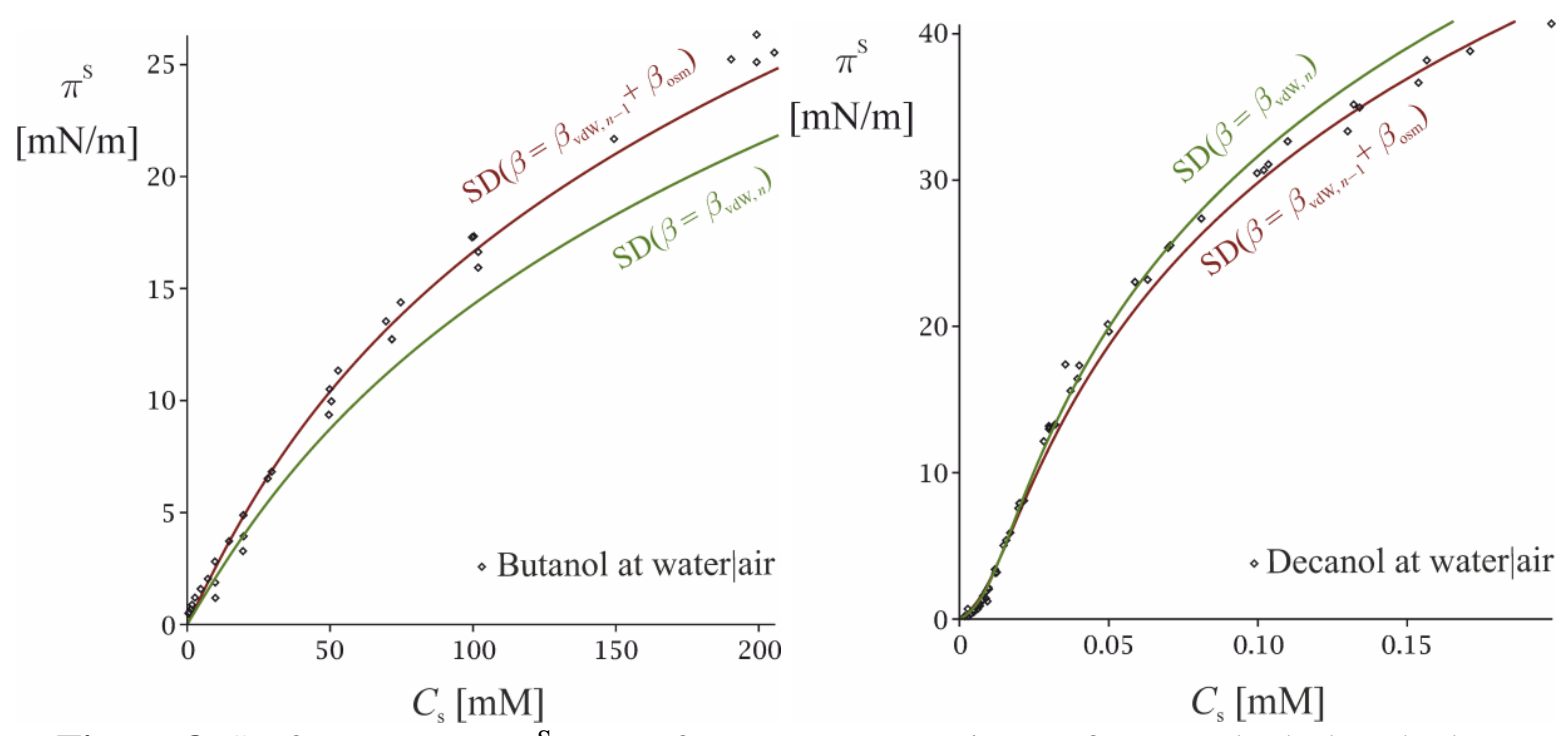

Figure 8. Surface pressure $\pi^{\mathrm{S}}$ vs. surfactant concentration $C_{\mathrm{s}}$ for two alcohols. The brown lines are the SD model with attraction parameter $\beta$ set to the sum of the effective osmotic attraction $\beta_{\text {osm }}$ and $\beta_{\mathrm{vdW}, n-1}$ corrected for one immersed methylene group, Eq (20). The computations are using the two fitting parameters of the whole homologous series:

$K_{\mathrm{a} 0}=16.9 \AA$ and $\beta_{\text {osm }}=0.98$ (Table 1). The green lines are with $\beta=\beta_{\mathrm{vdW}, n}$ (as in Ref. [18]); $K_{\mathrm{a} 0}=13.8 \AA$.

As mentioned in the main text, unlike the 1-component SD model, the model presented in this work allows the amount of solvent in the monolayer to be computed. From the value $\beta_{\text {osm }}$ $=0.98$ of the alcohol series and Eq (16), we obtain $\psi_{\mathrm{w} 0}=0.77$. We then use Eq (13) to compute $\psi_{\mathrm{w}}$ and Eqs (4)\&(5) to compute $C_{\mathrm{s}}$ for each $\psi_{\mathrm{s}}$. The results, in coordinates $\psi \mathrm{vs} . \lg C_{\mathrm{s}}$, are shown in Figure 9, for both butanol and decanol. The qualitative trends are similar to those for $\mathrm{C}_{12} \mathrm{H}_{25} \mathrm{EO}_{6}$ in Figure 7 (water is desorbed as more alcohol adsorbs; the monolayer is compressible and the total coverage increases with $C_{\mathrm{s}}$ ). The dependence is similar for the two alcohols but shifted to lower concentrations with the more surface-active decanol.

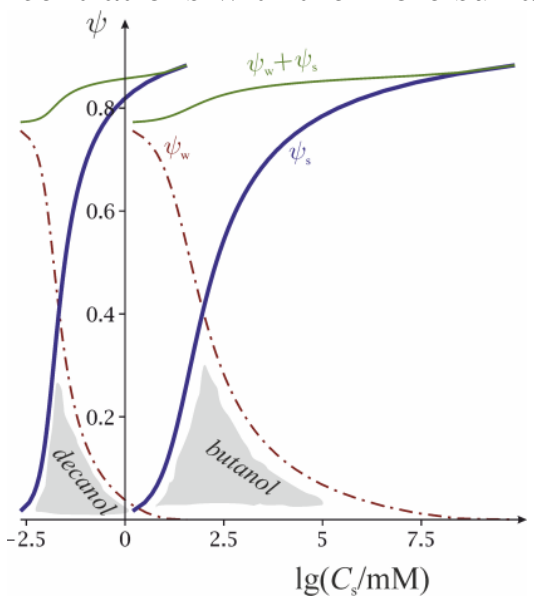

Figure 9. Surface coverage with solvent molecules $\left(\psi_{\mathrm{w}}=\alpha_{\mathrm{w}} \Gamma_{\mathrm{w}}\right)$, surfactant $\left(\psi_{\mathrm{s}}=\alpha_{\mathrm{s}} \Gamma_{\mathrm{s}}\right)$, and total $\left(\psi_{\mathrm{w}}+\psi_{\mathrm{s}}\right)$ vs. logarithm of surfactant concentration in the bulk phase for butanol and decanol.

In Figure 10, we give for completeness the comparison between the corrected SD model (with $\beta_{\text {osm }}$ and $K_{\mathrm{a} 0}$ from Table 1) and the experimental data for acids (from $\mathrm{C}_{2} \mathrm{H}_{5} \mathrm{COOH}$ to $\mathrm{C}_{9} \mathrm{H}_{19} \mathrm{COOH}$ ) from Refs. [10,60-64], used for the determination of $\beta_{\text {osm }}$ (see S6 for the full list of data). 


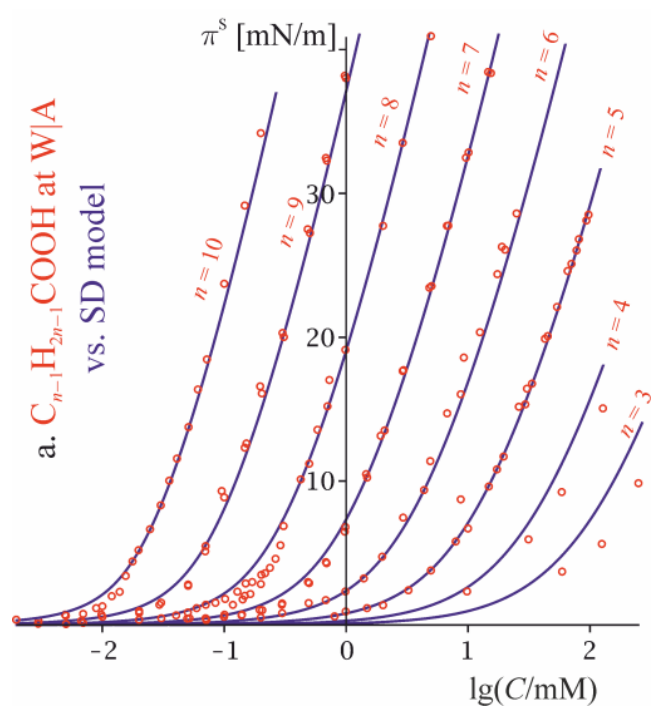

Figure 10. Surface pressure $\pi^{\mathrm{S}}$ vs. decimal logarithm of the concentration $C_{\mathrm{s}}$ of carboxylic acids at $\mathrm{W} \mid \mathrm{A}$ (low $\mathrm{pH}$ ). Lines stand for the SD model with attraction parameter $\beta=\beta_{\mathrm{osm}}+\beta_{\mathrm{vdW}, n-1}$. Two adjustable parameters for the whole homologous series were determined: $\ln \left(K_{\mathrm{a} 0} / \mathrm{m}\right)=-20.1$ and $\beta_{\text {osm }}=0.84$.

Let us also compare the results with directly measured adsorption of hexanol and $\mathrm{C}_{12} \mathrm{H}_{25} \mathrm{EO}_{6}$. Before doing that, we should mention that the adsorption Lu et al. [67] obtained via tensiometric data differs significantly from their neutron scattering results. To complicate things further, the surface tension measured by $\mathrm{Lu}$ et al. [67] for various $\mathrm{C}_{12} \mathrm{H}_{25} \mathrm{EO}_{6}$ concentrations is quite high compared to the data in Ref. $[9,12,65]$ we used for the same surfactant and temperature. The neutron scattering data are compared in Figure 11a with the SD model, Eqs (4)\&(5). The difference is within the experimental uncertainty of $8 \%$ (despite the systematic negative deviations of the data). The dashed line is the reorientation model of Fainerman and Miller (discussed in Sec. [3]). In Figure 11b, similar comparison is shown between the hexanol neutron scattering data of $\mathrm{Li}$ et al. [66] and the SD model. The deviations show, first of all, that the surface tension data we use do not agree very well with the neutron reflectivity data (the authors of the neutron scattering studies have shown themselves disagreement of a similar order of magnitude $[66,67])$. The reasons can be: (i) impurities in the commercially available $\mathrm{C}_{12} \mathrm{H}_{25} \mathrm{EO}_{6}$ [67]; (ii) depletion of the solution - the Teflon trough used in Refs. $[66,67]$ is relatively shallow $(5 \mathrm{~mm})$ and for the dilute $\mathrm{C}_{12} \mathrm{H}_{25} \mathrm{EO}_{6}$ solution, the material adsorbed at the water|air and the water|Teflon can be a significant fraction of the total $\mathrm{C}_{12} \mathrm{H}_{25} \mathrm{EO}_{6}$ in the system; (iii) in the case of $\mathrm{C}_{6} \mathrm{H}_{13} \mathrm{OH}$, some of the surfactant may be depleted from the solution through evaporation and adsorption/condensation at the glass/aluminium surface of the container (although the authors rule out this possibility [66]); (iv) slow kinetics of adsorption. 

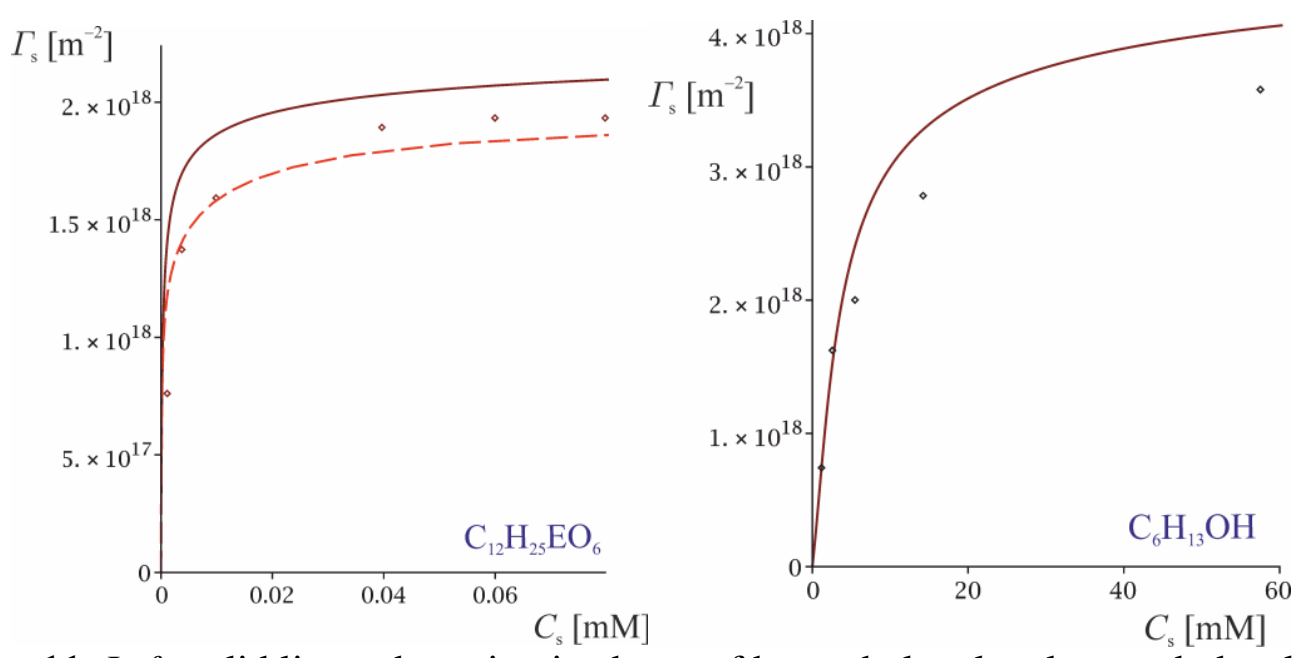

Figure 11. Left, solid line: adsorption isotherm of hexaethylenglycol monododecyl ether predicted by the SD model, Eqs (4)\&(5), with $\beta$ given by Eq (23) and the parameters in Table 1. Dashed line: the reorientation model of Fainerman and Miller. Points: neutron reflectivity data [67]. Right: the adsorption isotherm of hexanol according to the SD model with $\beta$ given by Eq (23) and the parameters in Table 1 (line) against neutron reflectivity data [66].

The values of the lateral attraction parameters of all surfactants are summarized in Table 2, as obtained via numerical integration of Eq (23). The results are compared with the previous model for the lateral attraction from Ref. [23]. 
Table 2. Values of $\beta$ of the simple SD model $\left(\beta_{\mathrm{vdW}, n}\right)$ and of the one corrected for the presence of solvent in the monolayer and for the immersed $\mathrm{CH}_{2}$ group $\left(\beta_{\mathrm{vdW}, n-1}+\beta_{\mathrm{osm}}\right.$, Eq (23)) for the three homologous series studied.

\begin{tabular}{|c|c|c|c|c|c|c|}
\hline & \multicolumn{2}{|c|}{$\mathrm{C}_{n} \mathrm{H}_{2 n+1} \mathrm{OH}, 21^{\circ} \mathrm{C}$} & \multicolumn{2}{c|}{$\mathrm{C}_{n-1} \mathrm{H}_{2 n-1} \mathrm{COOH}, 21^{\circ} \mathrm{C}$} & \multicolumn{2}{c|}{$\mathrm{C}_{n} \mathrm{H}_{2 n+1} \mathrm{EO}_{6}, 25^{\circ} \mathrm{C}$} \\
\hline$\alpha_{\mathrm{s}}=$ & \multicolumn{2}{|c|}{${ }^{\mathrm{i}} 16.5 \AA^{2}$} & \multicolumn{2}{|c|}{${ }^{\mathrm{i}} 18 \AA^{2}$} & $\begin{array}{c}33.3 \\
\AA^{2}\end{array}$ & $\begin{array}{c}34.3 \\
\AA^{2}\end{array}$ \\
\hline$n$ & $\beta_{\mathrm{vdW}, n}$ & $\begin{array}{c}\beta_{\mathrm{vdW}, n-1} \\
+\beta_{\mathrm{osm}}\end{array}$ & $\beta_{\mathrm{vdW}, n}$ & $\begin{array}{c}\beta_{\mathrm{vdW}, n-1} \\
+\beta_{\mathrm{osm}}\end{array}$ & $\beta_{\mathrm{vdW}, n}$ & $\begin{array}{c}\beta_{\mathrm{vdW}, n-1} \\
+\beta_{\mathrm{osm}}\end{array}$ \\
\hline 3 & 1.01 & 1.43 & 0.75 & 1.18 & & \\
\hline 4 & 1.80 & 1.99 & 1.32 & 1.59 & & \\
\hline 5 & 2.89 & 2.78 & 2.06 & 2.16 & & \\
\hline 6 & 4.35 & 3.87 & 3.00 & 2.90 & & \\
\hline 7 & 6.32 & 5.33 & 4.18 & 3.84 & & \\
\hline 8 & 9.01 & 7.30 & 5.68 & 5.02 & & \\
\hline 9 & 12.7 & 9.99 & 7.59 & 6.52 & & \\
\hline 10 & 18.0 & 13.7 & 10.06 & 8.43 & & \\
\hline 11 & $25.5^{\mathrm{e}}$ & $19.0^{\mathrm{e}}$ & $13.3^{\mathrm{e}}$ & $10.9^{\mathrm{e}}$ & & \\
\hline 12 & $36.2^{\mathrm{e}}$ & $26.4^{\mathrm{e}}$ & $17.5^{\mathrm{e}}$ & $14.1^{\mathrm{e}}$ & 1.16 & 1.43 \\
\hline 13 & $52.0^{\mathrm{e}, \mathrm{pt}}$ & $37.2^{\mathrm{e}}$ & $23.1^{\mathrm{e}}$ & $18.3^{\mathrm{e}}$ & $1.31^{\mathrm{e}}$ & $1.56^{\mathrm{e}}$ \\
\hline 14 & & $52.9^{\mathrm{e}, \mathrm{pt}}$ & $30.6^{\mathrm{e}}$ & $24.0^{\mathrm{e}}$ & 1.47 & 1.69 \\
\hline & & & $40.8^{\mathrm{e}, \mathrm{pt}}$ & $31.5^{\mathrm{e}}$ & & \\
\hline & & & & $41.6^{\mathrm{e}, \mathrm{pt}}$ & & \\
\hline
\end{tabular}

${ }^{\mathrm{pt}}$ Surfactants below their critical point displaying a gaseous-LE phase transition according to the analysis by the law of corresponding states in Ref. [23]. ${ }^{\text {e }}$ Values are extrapolated or interpolated (not based on experimental data for the respective homologue). ${ }^{i}$ Area following from crystallographic data and collapse of insoluble monolayers [23]. ${ }^{\text {ii }}$ Area obtained as a fitting parameter from the data for $\mathrm{C}_{n} \mathrm{H}_{2 n+1} \mathrm{EO}_{6}$, cf. Table 1.

\section{S3. Limit of the adsorption isotherms at $\psi_{w} \rightarrow 0$ (dry monolayer)}

An explicit dependence of the surface fraction of solvent on the surface fraction of surfactant can be obtained by expanding Eq (13) in series at $\psi_{\mathrm{w}} \rightarrow 0$ :

$$
\psi_{\mathrm{w}}=\frac{\psi_{\mathrm{w} 0}}{1-\psi_{\mathrm{w} 0}} \mathrm{e}^{\frac{\psi_{\mathrm{w} 0}\left(3-2 \psi_{\mathrm{w} 0}\right)}{\left(1-\psi_{\mathrm{w} 0}\right)^{2}}}\left(1-\psi_{\mathrm{s}}\right) \mathrm{e}^{-\frac{\psi_{\mathrm{s}}\left[\frac{2}{\sqrt{r}}\left(1-\psi_{\mathrm{s}}\right)+\frac{1}{r}\right]}{\left(1-\psi_{\mathrm{s}}\right)^{2}}} ;
$$

this asymptote holds true for dense monolayers. The adsorption isotherm of the surfactant in the same limit follows from $\mathrm{Eq}(14)$ :

$$
\alpha_{\mathrm{s}} K_{\mathrm{a}}^{\prime} C_{\mathrm{s}}=\frac{\psi_{\mathrm{s}}}{1-\psi_{\mathrm{s}}} \mathrm{e}^{\frac{\psi_{\mathrm{s}}\left(3-2 \psi_{\mathrm{s}}\right)}{\left(1-\psi_{\mathrm{s}}\right)^{2}}}, \text { where } K_{\mathrm{a}}^{\prime}=\frac{K_{\mathrm{a}}}{1-\psi_{\mathrm{w} 0}} \mathrm{e}^{\frac{\psi_{\mathrm{w} 0}\left[2 \sqrt{r}\left(1-\psi_{\mathrm{w} 0}\right)+r\right]}{\left(1-\psi_{\mathrm{w} 0}\right)^{2}}} .
$$

This result is formally equivalent to the HFL isotherm, apart from the adsorption constant which contains an osmotic contribution from the solvent expulsed from the monolayer. These results are hardly relevant for W|A surface, as the exponential dependence (33) is close to the exact Eq (13) only at rather dense monolayers, e.g., $\psi_{\mathrm{s}}>0.7$. Still, they may be useful in the analysis of the adsorption at the surface of non-aqueous media. 


\section{S4. The case of concentrated bulk solution}

If the concentration of surfactant in the bulk solution is high enough, then the chemical potential of the water changes with $C_{\mathrm{s}}$. In this case, the adsorption isotherm of the solvent has to be modified to include the change in activity of the bulk solvent:

$$
\gamma_{\mathrm{w}} \psi_{\mathrm{w}}=\gamma_{\mathrm{w} 0} \psi_{\mathrm{w} 0} \exp \left(-\gamma_{\mathrm{w}}^{\mathrm{B}} M_{\mathrm{w}} C_{\mathrm{m}}\right) ;
$$

here, $\gamma_{\mathrm{w}}^{\mathrm{B}}\left(C_{\mathrm{m}}\right)$ is the molality-based osmotic coefficient of the bulk solution, and $M_{\mathrm{w}}$ is the molar mass of the solvent; $C_{\mathrm{m}}$ is the molal concentration of the surfactant (which is more convenient than molarity $C_{\mathrm{s}}$ for concentrated solutions of variable density). For dilute solutions, $\gamma_{\mathrm{w}}^{\mathrm{B}} \rightarrow 1$, and $M_{\mathrm{w}} C_{\mathrm{m}}$ is small, so the equilibrium condition simplifies to $\gamma_{\mathrm{w}} \psi_{\mathrm{w}}=\gamma_{\mathrm{w} 0} \psi_{\mathrm{w} 0}$ (leading to Eq (13)). None of the surfactants we consider requires this correction as $1 \gg M_{\mathrm{w}} C_{\mathrm{m}}$ for the concentration ranges we studied, cf. the supplementary Excel spreadsheet. The correction would become important for, e.g., concentrated methanol and ethanol in water.

The respective adsorption isotherm of the surfactant reads:

$$
\alpha_{\mathrm{s}} K_{\mathrm{a}, \mathrm{m}} \gamma_{\mathrm{s}}^{\mathrm{B}} C_{\mathrm{m}}=\gamma_{\mathrm{s}} \psi_{\mathrm{s}},
$$

where $K_{\mathrm{a}, \mathrm{m}}\left[\mathrm{kg} / \mathrm{m}^{2}\right]$ is the molality-based adsorption constant $\left(R T \ln K_{\mathrm{a}, \mathrm{m}}\right.$ is the adsorption energy with respect to ideal standard states of $1 \mathrm{~mol} / \mathrm{m}^{2}$ adsorbed surfactant at the surface and $1 \mathrm{~mol} / \mathrm{kg}$ surfactant in the bulk); $\gamma_{\mathrm{s}}^{\beta}\left(C_{\mathrm{m}}\right)$ is the activity coefficient of the surfactant. The osmotic and the activity coefficients are related via the integral form of the Gibbs-Duhem relation:

$$
\gamma_{\mathrm{w}}^{\mathrm{B}}=1+\frac{1}{C_{\mathrm{m}}} \int_{0}^{C_{\mathrm{m}}} C_{\mathrm{m}} \frac{\mathrm{d} \ln \gamma_{\mathrm{s}}^{\mathrm{B}}}{\mathrm{d} C_{\mathrm{m}}} \mathrm{d} C_{\mathrm{m}} .
$$

As usually with dilute solutions, the activity coefficient of the surfactant in Eq (36) becomes important at much lower concentration than the osmotic coefficient of the solvent in Eq (35). For this reason, we had to take into account the activity of propanol and we did not consider tensiometric data for concentration of many surfactants above an estimated threshold concentration above which $\gamma_{s}^{\beta}$ becomes significantly different from 1 (cf. the supplementary Excel spreadsheet S6).

Two final modifications of the general theory of adsorption are required with concentrated solutions. The first one is that the Gibbs adsorption $\Gamma_{\mathrm{s} \text {,eqm }}$ is different from the density $\Gamma_{\mathrm{s}}$ of the surfactant in the monolayer, cf. Eq (32). The second is that the change of the osmotic pressure in the bulk has to be accounted for when computing the surface pressure (Eq (9) gives the surface pressure only under the assumption for negligible change of the osmotic pressure in the bulk with the addition of surfactant).

\section{S5. Derivation of the activity coefficients of the LHP model}

$\mathrm{Eq}(10)$ is equivalent to the following system of differential equations:

$$
\begin{aligned}
& \frac{\partial}{\partial \psi_{\mathrm{w}}} \frac{\alpha_{\mathrm{s}} \pi^{\mathrm{s}}\left(\psi_{\mathrm{s}}, \psi_{\mathrm{w}}\right)}{k_{\mathrm{B}} T}=r \psi_{\mathrm{w}} \frac{\partial \ln \left(\gamma_{\mathrm{w}}\left(\psi_{\mathrm{s}}, \psi_{\mathrm{w}}\right) \psi_{\mathrm{w}}\right)}{\partial \psi_{\mathrm{w}}}+\psi_{\mathrm{s}} \frac{\partial \ln \left(\gamma_{\mathrm{s}}\left(\psi_{\mathrm{s}}, \psi_{\mathrm{w}}\right) \psi_{\mathrm{s}}\right)}{\partial \psi_{\mathrm{w}}} ; \\
& \frac{\partial}{\partial \psi_{\mathrm{s}}} \frac{\alpha_{\mathrm{s}} \pi^{\mathrm{s}}\left(\psi_{\mathrm{s}}, \psi_{\mathrm{w}}\right)}{k_{\mathrm{B}} T}=r \psi_{\mathrm{w}} \frac{\partial \ln \left(\gamma_{\mathrm{w}}\left(\psi_{\mathrm{s}}, \psi_{\mathrm{w}}\right) \psi_{\mathrm{w}}\right)}{\partial \psi_{\mathrm{s}}}+\psi_{\mathrm{s}} \frac{\partial \ln \left(\gamma_{\mathrm{s}}\left(\psi_{\mathrm{s}}, \psi_{\mathrm{w}}\right) \psi_{\mathrm{s}}\right)}{\partial \psi_{\mathrm{s}}},
\end{aligned}
$$

where $\pi^{\mathrm{S}}$ is given by Eq (9). These are two partial differential equations (two dimensional, variables $\psi_{\mathrm{s}}$ and $\psi_{\mathrm{w}}$ ) for two unknown functions: the activity coefficients $\gamma_{\mathrm{s}}$ and $\gamma_{\mathrm{w}}$. The 
functions have to fulfil also two boundary conditions - in infinitely dilute monolayer, both activity coefficients have to be equal to 1 , i.e.

$$
\gamma_{\mathrm{w}}\left(\psi_{\mathrm{s}}=0, \psi_{\mathrm{w}}=0\right)=\gamma_{\mathrm{s}}\left(\psi_{\mathrm{s}}=0, \psi_{\mathrm{w}}=0\right)=1 \text {. }
$$

The solution for $\gamma_{\mathrm{w}}$ and $\gamma_{\mathrm{s}}$ to Eqs (38)\&(39) is given by Eqs (11). This can be readily demonstrated by substitution of Eqs (9)\&(11) into Eqs (38) - the execution of the differential operations leads to an identity. 